\title{
Horton laws for hydraulic-geometric variables and their scaling exponents in self-similar Tokunaga river networks
}

\author{
V. K. Gupta ${ }^{1}$ and O. J. Mesa ${ }^{2}$ \\ ${ }^{1}$ Department of Civil, Environmental and Architectural Engineering and Cooperative Institute for Research in Environmental \\ Sciences, University of Colorado, Boulder, Colorado, USA \\ ${ }^{2}$ Departamento de Geociencias y Medio Ambiente, Universidad Nacional de Colombia, Medellín, Colombia
}

Correspondence to: V. K. Gupta (vijay.gupta@colorado.edu)

Received: 4 March 2014 - Published in Nonlin. Processes Geophys. Discuss.: 16 April 2014

Revised: 1 August 2014 - Accepted: 20 August 2014 - Published: 30 September 2014

\begin{abstract}
An analytical theory is developed that obtains Horton laws for six hydraulic-geometric $(\mathrm{H}-\mathrm{G})$ variables (stream discharge $Q$, width $W$, depth $D$, velocity $U$, slope $S$, and friction $n^{\prime}$ ) in self-similar Tokunaga networks in the limit of a large network order. The theory uses several disjoint theoretical concepts like Horton laws of stream numbers and areas as asymptotic relations in Tokunaga networks, dimensional analysis, the Buckingham Pi theorem, asymptotic self-similarity of the first kind, or SS-1, and asymptotic self-similarity of the second kind, or SS-2. A self-contained review of these concepts, with examples, is given as "methods". The H-G data sets in channel networks from three published studies and one unpublished study are summarized to test theoretical predictions. The theory builds on six independent dimensionless river-basin numbers. A mass conservation equation in terms of Horton bifurcation and discharge ratios in Tokunaga networks is derived. Assuming that the $\mathrm{H}-\mathrm{G}$ variables are homogeneous and self-similar functions of stream discharge, it is shown that the functions are of a power law form. SS-1 is applied to predict the Horton laws for width, depth and velocity as asymptotic relationships. Exponents of width and the Reynolds number are predicted and tested against three field data sets. One basin shows deviations from theoretical predictions. Tentatively assuming that SS-1 is valid for slope, depth and velocity, corresponding Horton laws and the $\mathrm{H}-\mathrm{G}$ exponents are derived. Our predictions of the exponents are the same as those previously predicted for the optimal channel network (OCN) model. In direct contrast to our work, the OCN model does not consider Horton laws for the $\mathrm{H}-\mathrm{G}$ variables, and uses optimality assumptions. The predicted exponents deviate substantially
\end{abstract}

from the values obtained from three field studies, which suggests that $\mathrm{H}-\mathrm{G}$ in networks does not obey SS-1. It fails because slope, a dimensionless river-basin number, goes to 0 as network order increases, but, it cannot be eliminated from the asymptotic limit. Therefore, a generalization of SS-1, based on SS-2, is considered. It introduces two anomalous scaling exponents as free parameters, which enables us to show the existence of Horton laws for channel depth, velocity, slope and Manning friction. These two exponents are not predicted here. Instead, we used the observed exponents of depth and slope to predict the Manning friction exponent and to test it against field exponents from three studies. The same basin mentioned above shows some deviation from the theoretical prediction. A physical reason for this deviation is given, which identifies an important topic for research. Finally, we briefly sketch how the two anomalous scaling exponents could be estimated from the transport of suspended sediment load and the bed load. Statistical variability in the Horton laws for the $\mathrm{H}-\mathrm{G}$ variables is also discussed. Both are important open problems for future research.

\section{Introduction}

Several key papers have been published on self-similar river networks in the last twenty years. As a sample, see Tokunaga (1978), Peckham (1995b), Peckham and Gupta (1999), Dodds and Rothman (1999), Veitzer and Gupta (2000), Troutman (2005), Veitzer et al. (2003), McConnell and Gupta (2008). Tokunaga model among them is very important because it is mathematically simple and it predicts many 
topological and geometrical features that are observed in real channel networks (Tokunaga, 1978; Peckham, 1995a). The predictions are made in terms of the Horton laws that are explained in Sect. 2 on the background. Our paper develops an analytical theory to predict Horton relationships for six hydraulic-geometric $(\mathrm{H}-\mathrm{G})$ variables (stream discharge $Q_{\omega}$, width $W_{\omega}$, depth $D_{\omega}$, velocity $U_{\omega}$, slope $S_{\omega}$, and Manning friction $n_{\omega}^{\prime}$ ) in self-similar Tokunaga river networks. It is the first study that generalizes the theory from topology and geometry to $\mathrm{H}-\mathrm{G}$ in channel networks. The $\mathrm{H}-\mathrm{G}$ exponents for $W_{\omega}$, Reynolds number, $\Pi_{5}(\omega)$, and $n_{\omega}^{\prime}$ are predicted and tested against observed exponents from three field studies. Developing an analytical theory of $\mathrm{H}-\mathrm{G}$ in channel networks is a long-standing, fundamental open problem in Hydro-geomorphology that is addressed here. Next we explain the significance of this work.

Prediction of floods in river basins with sparse or no streamflow data is a hydrologic engineering problem of great practical significance. Known as regional flood frequency analysis, it has over 100 years of history (Dawdy et al., 2012). An acronym for this problem that is widely used is prediction in ungauged basins (PUB) (Dawdy, 2007; Sivapalan et al., 2003). PUB is common to both developing as well as industrialized countries. A nonlinear geophysical theory is being developed for almost 30 years to solve the PUB problem for floods. Two review articles have been published on this theory (Gupta and Waymire, 1998; Gupta et al., 2007). Given space-time rainfall intensity field for any rainfallrunoff event, the theory attempts to predict stream flow hydrographs at all the "junctions" (where two or three channels meet) in a channel network. The theory requires a model to transform rainfall to runoff in space and time in a basin (Furey et al., 2013), and space-time river flow dynamics in a network (Mantilla, 2007). Modeling of flow dynamics requires a theory of $\mathrm{H}-\mathrm{G}$ in a channel network, because practically no such data sets exist. Our paper begins to fill a longstanding need in this context.

By extending the Horton laws to $\mathrm{H}-\mathrm{G}$ variables, our paper shows how geomorphology, hydrology and channel hydraulics are linked in river networks. Consequently, it opens a new door to understanding how the geometry, statistics and dynamics in river networks are mutually coupled on many spatial scales, which has far-reaching implications for understanding and modeling river flows, as explained above, and transport of sediments and pollutants in river networks in the future. A few discrete research efforts have been made to understand the nature of the flood scaling from physical processes on an annual timescale (Poveda et al., 2007; Lima and Lall, 2010), as well as on the event timescale (Ogden and Dawdy, 2003), but connecting the body of work on the annual scale to flood scaling for events remains an important open problem (Gupta et al., 2010).

Our paper is organized as follows. Section 2 gives a brief review of the literature. It also includes two "method" sections that give a self-contained review of analytical meth- ods used in the theory. Section 2.1 contains a review of the Horton laws for network topology and geometry as asymptotic relations in self-similar Tokunaga networks that are taken from McConnell and Gupta (2008). Section 2.2 gives a review of similarity or similitude that is based on dimensional analysis and that explains the Buckingham Pi theorem. It is followed by a review of "asymptotic self-similarity of the first kind", or SS-1 for short. In many cases including the present case, SS-1 requires a generalization involving "asymptotic self-similarity of the second kind", or SS-2 for short. Physical examples are given to explain these methodological concepts.

Section 3 explains the $\mathrm{H}-\mathrm{G}$ data sets in channel networks from three published studies and one unpublished study used in this paper to test theoretical predictions. In Sect. 4, an application of the Buckingham Pi theorem gives a total of six independent dimensionless river-basin numbers. The six numbers are specified using physical arguments rather than formal dimensional analysis. In Sect. 5, we formulate a mass conservation equation for a river network indexed by Strahler order. We apply the results from Sect. 2.1 to obtain a solution of this equation in terms of Horton bifurcation, area and discharge ratios in the limit of large network order $\Omega$. It applies to small order streams, $\omega=1,2,3, \ldots$.

In Sect. 6, we consider three $\mathrm{H}-\mathrm{G}$ variables, $W_{\omega}, D_{\omega}$ and $U_{\omega}$, and show that they are power-law functions of discharge. By definition, $Q_{\omega}=U_{\omega} W_{\omega} D_{\omega}$. Horton laws are obtained as asymptotic relations for these three $\mathrm{H}-\mathrm{G}$ variables. We show that self-similar solutions involving SS-1 hold asymptotically for the width and the Reynolds number. Values of their $\mathrm{H}-\mathrm{G}$ exponents are predicted and tested against field data from Sect. 3.

In Sect. 7, it is tentatively assumed that the SS-1 framework from Sect. 6 is valid for $S_{\omega}, D_{\omega}$ and $U_{\omega}$. Horton laws for these three $\mathrm{H}-\mathrm{G}$ variables are derived asymptotically, and their exponents are predicted. Our predictions of the exponents are the same as those previously predicted by Rodríguez-Iturbe et al. (1992) for the optimal channel network (OCN) model. In direct contrast to our work, the OCN model does not consider Horton laws for these $\mathrm{H}-\mathrm{G}$ variables, and uses optimality assumptions. In this sense, the foundations of our theory based on self-similarity and dimensional analysis are very different from those of the OCN model. The deviations between the observed and predicted exponents are substantial, suggesting that $\mathrm{H}-\mathrm{G}$ in networks does not obey SS-1.

In Sect. 8, we explain that the reason for the failure of SS-1 is that the slope, one of the dimensionless numbers, goes to 0 as the network order increases, but slope cannot be eliminated from the asymptotic limit. Therefore, a generalization of SS-1 requiring SS-2 is needed. This section is divided into four subsections. The first one introduces two anomalous scaling exponents, $\alpha$ and $\beta$, in the theory. It enables us to show the existence of Horton laws for channel depth, velocity, and slope, and derive expressions for their 
exponents as functions of $\alpha$ and $\beta$. These two scaling exponents are free parameters, which cannot be predicted from dimensional considerations. To make progress with developing and testing the theory, we consider the Manning friction coefficient as the fifth $\mathrm{H}-\mathrm{G}$ variable in the second subsection, which can be estimated from values of slope and velocity. We predict a Horton law for the Manning friction coefficient and derive an expression for its exponent. The third subsection tests the prediction of the exponent against three field studies. One of these basins shows some deviation from the theoretical prediction. A physical reason for this deviation is investigated in the fourth subsection.

Two fundamental physical processes that shape the $\mathrm{H}-\mathrm{G}$ of channels are transport of the suspended sediment load and transport of the bed load, which are not considered here. In Sect. 9, we sketch in a preliminary manner how these two physical processes could be used to determine the two anomalous scaling exponents. Inclusion of statistical variability in the Horton laws for the $\mathrm{H}-\mathrm{G}$ variables is also discussed. Both are important open problems for future research. The paper is concluded in Sect. 10.

\section{Background and methods}

Leopold and Maddock (1953) first introduced the hydraulic geometry $(\mathrm{H}-\mathrm{G})$ of rivers "at-a-station" and in the "downstream direction". "At-a-station" $\mathrm{H}-\mathrm{G}$ relations refer to temporal variability of width, depth, velocity, slope, channel roughness, and sediment transport as functions of discharge, and "downstream" $\mathrm{H}-\mathrm{G}$ relations refer to their spatial variability as functions of discharge. An extensive literature has developed on these topics; see Singh (2003) for a recent review of the literature. This body of literature, though important, is not directly relevant to the objectives of our paper. Indeed, Singh (2003) concluded his review paper with the statement, "the work on hydraulic geometry of channels serves as an excellent starting point to move on to the development of a theory of drainage basin geometry and channel network evolution. This will permit integration of channel hydraulics and drainage basin hydrology and geomorphology".

In a classic paper, Leopold and Miller (1956) extended the $\mathrm{H}-\mathrm{G}$ relations to drainage networks. A brief review of pertinent concepts required to understand this work follows. Horton (1945) first discovered "Horton laws" in quantitative geomorphology with the aid of maps. The original motivation was to define stream size based on a hierarchy of tributaries. The most common method for defining a spatial scale in a hierarchical branched network is the method of Horton-Strahler ordering, or Strahler ordering for short, because Strahler $(1952,1957)$ modified the ordering system that Horton had introduced. Strahler ordering assigns, $\omega=1$ to all the unbranched streams. They contain the highest level of spatial resolution for a network and thereby define a spatial scale. Continuing downstream through the network, where two streams of identical order $\omega$ meet, they form a stream of order $\omega+1$. Where two streams of different orders meet, the downstream channel is assigned the higher of the two orders. This continues throughout the network, labeling each stream, and ending with the stream of order $\Omega$. By definition, any network contains only one stream of order $\Omega$ called the network order. Strahler ordering defines a one-to-one map under pruning; i.e., if the streams of order 1 are pruned and the entire tree is renumbered, the order 2 streams identically become the new order 1 streams, the order 3 streams become order 2, and so on throughout the network. The order of the entire network decreases by one. It is a necessary condition for defining self-similarity for a hierarchical branched network that is reviewed in Sect. 2.1.

Strahler ordering led to the discovery of the "Horton laws of drainage composition". They are often referred to simply as the Horton laws. The most famous of the Horton laws is the law of stream numbers for $N_{\omega}$, denoting the number of streams of order $\omega$ in a network of order $\Omega$. It is traditionally written as

$\frac{N_{\omega}}{N_{\omega+1}}=R_{B}, 1 \leq \omega \leq \Omega$.

The number $R_{B}$ is called the bifurcation ratio. Observations from real river networks show a limited range of $R_{B}$ values between three and five. These are not formal laws because they have not been proved from first principles, however, they are widely observed in real river networks. Similar relationships are observed for lengths, slopes, and areas.

Shreve (1967) developed the "random model" of channel networks and thereby offered the first theoretical understanding of Eq. (1). He introduced the basic topological concepts of channel links (defined as the segment of channel between two adjacent junctions where two or three channels meet) and magnitude (defined as the total number of non-branching or source channel links). Shreve (1967) showed that in the limit as magnitude goes to infinity, $R_{B}$ converges to 4 . This demonstration showed for the first time that the empirical Eq. (1) can be derived as an asymptotic result from a suitable mathematical model of channel networks. We review this foundational issue in greater detail in the next subsection using the mathematical model of self-similar Tokunaga river networks.

Leopold and Miller (1956) extended the Horton laws to $\mathrm{H}-\mathrm{G}$ variables. Their reasoning was that channel discharge varies as a function of drainage area as a power law, $Q=$ $k A^{c}$. At the time, the Horton law for drainage area was known (Jarvis and Woldenberg, 1984). They tested the Horton law for discharge, and asserted that the Horton laws hold for the entire suite of $\mathrm{H}-\mathrm{G}$ variables as functions of discharge, e.g., width, depth, velocity, slope, channel roughness, and sediment transport. Until this paper was published, the Horton laws had been observed for only the topologic and geometric variables (Jarvis and Woldenberg, 1984). By extending the Horton laws to $\mathrm{H}-\mathrm{G}$ variables, the Leopold and 
Miller (1956) paper showed how river basin geomorphology, hydrology and channel hydraulics are linked. Consequently, it opened a new door to understanding how the geometry, statistics and dynamics in river networks are mutually coupled on many spatial scales, which has far-reaching implications for understanding and modeling river flows and sediment transport in river networks. This major objective has not been realized because the theoretical underpinning of the Horton laws and the $\mathrm{H}-\mathrm{G}$ exponents in channel networks has remained elusive. It remains a fundamental, longstanding open problem in Hydro-geomorphology that is addressed here.

The Strahler ordering and the Horton law concepts had a big impact on several areas, for example, (i) in modeling growth of plants and other hierarchical biological structures such as animal respiratory and circulatory systems, (ii) in the order of register allocation for compilation of high level programming languages, and (iii) in the analysis of social networks (Jarvis and Woldenberg, 1984; Pries and Secomb, 2011; Viennot and Vauchaussade de Chaumont, 1985; Park, 1985; Horsfield, 1980; Borchert and Slade, 1981; Berry and Bradley, 1976). The widespread appearance of Horton laws suggests that perhaps a "fundamental principle" underlies them. Indeed, recent research has shown that Horton laws are asymptotic relations that have been proved in theoretical self-similar river network models. Self-similarity is a form of scale invariance. Specifically, we have selected the selfsimilar Tokunaga network model to develop the theory in this paper. This model is briefly reviewed in the next Sect. 2.1.

An attempt to predict the $\mathrm{H}-\mathrm{G}$ exponents in river networks without the Horton laws is the theory of optimal channel networks (OCN) (Rodríguez-Iturbe et al., 1992). OCNs have been analytically shown to produce three universality classes in terms of scaling exponents, but none of these predictions agrees with the data (Maritan et al., 1996). Two comprehensive field programs were carried out in New Zealand (NZ) to test the OCN predictions (Ibbitt et al., 1998; McKerchar et al., 1998). However, the observed values of the $\mathrm{H}-\mathrm{G}$ exponents substantially deviated from the OCN predictions that is explained in greater detail in Sects. 7 and 8.3. Other attempts building on optimality ideas have used data from these two New Zealand basins (Molnar and Ramirez, 2002), but a foundational understanding of the geophysical origins of Horton laws for the $\mathrm{H}-\mathrm{G}$ variables and their exponents has remained elusive.

West et al. (1997) recently tackled a somewhat similar problem in the allometric theory of biological networks. Our treatment of the $\mathrm{H}-\mathrm{G}$ problem has some similarities but major differences with their approach. For example, West et al. (1997) appeal to an "optimality assumption" by maximizing or minimizing a function. By contrast the present theory uses no optimality assumption, but uses "self-similarity" as its basic building block.

The complexity resulting from space-time variability in climate, hydrology and lithology can be contrasted with the empirical observations like the Horton laws that suggest regularities related to similarity across scales, or selfsimilarity. We briefly review key results for the self-similar Tokunaga model in the next Subsection regarding Horton laws of stream numbers and magnitudes.

\subsection{Method: a brief review of self-similar Tokunaga river networks}

Eiji Tokunaga introduced the Tokunaga model (Tokunaga, 1978). It is based on Strahler ordering and involves the concept of self-similar topology in its construction. Unlike the random model of Shreve (1967), this model is deterministic and does not include any statistical variability that is observed in real networks. Therefore, the link lengths are assumed to be equal to 1 throughout the paper. Peckham (1995a) gave empirical evidence that supports the idea that the average topologic features of medium to large river networks can be well described by the Tokunaga model. For example, in real networks, generally $3<R_{B}<5$ that the Tokunaga model can exhibit but the random model predicts $R_{B}=4$ as mentioned above. Moreover, the mathematics in the Tokunaga model is simplified that is necessary to make progress in the $\mathrm{H}-\mathrm{G}$ problem addressed here.

The key building block of the model is a generator for side tributaries, $T_{\omega, \omega-k}$, which may be interpreted as the mean number of streams of order $\omega-k$ joining streams of order $\omega$ since real networks exhibit statistical variability in their branching structures (topology). Self-similarity in the network topology is reflected in the assumption that $T_{\omega, \omega-k}=$ $T_{k}, k=2,3, \ldots$. Tokunaga further restricted his model by requiring that $T_{k} / T_{k-1}=c, k=2,3, \ldots$, and $T_{1}=a$ where $c$ and $a$ are constant parameters associated with network topology. It leads to the generator expression

$T_{k}=a c^{k-1}, \quad k=1,2, \ldots$,

representing mean self-similar Tokunaga trees (Dodds and Rothman, 1999). These parameters are observable quantities in natural basins.

Predictions are based on a fundamental recursion equation governing $N_{k}$, the number of streams of order $k$,

$N_{k}=2 N_{k+1}+\sum_{j=1}^{\Omega-k} T_{j} N_{k+j}$.

McConnell and Gupta (2008) proved that the ratio $N_{k} / N_{k+1}$ converges to $R_{B}$ in the limit as $\Omega-k \rightarrow \infty$. The limit applies to small-order streams, $k=1,2, \ldots$, as the network order $\Omega \rightarrow \infty$. McConnell and Gupta (2008) gave a physical interpretation of the limit as follows: "note that if the overall order $\Omega$ could be increased, we would expect to see more streams of higher and lower orders. However, when all the streams of smaller orders $k$ and $k+1$ in a basin of very large order $\Omega$ are counted, we expect that we have captured a significant and representative portion of the side tributaries, and 
the observed bifurcation ratio more closely matches the predicted ratio. This physical argument clarifies the use of the limit, $\Omega-k \rightarrow \infty$ ". The solution to Eq. (3) is given by

$$
R_{B}=\frac{(2+a+c)+\sqrt{(2+a+c)^{2}-8 c}}{2} .
$$

McConnell and Gupta (2008) also proved a Horton law for stream magnitudes, $M_{\omega}, \omega=1,2, \ldots$ from the above result. Magnitude is defined in Sect. 2, and is the topological equivalent of drainage area. The Horton law for magnitudes is expressed as

$\lim _{\Omega-\omega \rightarrow \infty} \frac{M_{\omega+1}}{M_{\omega}}=R_{M}=\lim _{\Omega-\omega \rightarrow \infty} \frac{A_{\omega+1}}{A_{\omega}}=R_{A}$,

where the Horton magnitude ratio $R_{M}$ equals $R_{B}$ and $R_{A}$, and $R_{A}$ is the Horton area ratio. We remark that the random model obeys self-similarity in a mean sense, and it has $a=1$ and $c=2$. Equation (4) predicts $R_{B}=4$, which agrees with the random model (Shreve, 1967).

Finally, we review two results, which were previously reported by others, and which have been shown to hold for Tokunaga networks. The topological fractal dimension $D_{\mathrm{T}}$ for Tokunaga networks is given by $D_{\mathrm{T}}=\log R_{B} / \log R_{C}$, where $R_{B}$ is the Horton bifurcation ratio and $R_{C}$ is the link ratio (Peckham, 1995a). Since $R_{B}=R_{A}$, and link lengths are constant, and as explained at the beginning, $R_{C}=R_{L}$, it follows that $R_{L}=R_{A}^{1 / D_{\mathrm{T}}}$, where $R_{L}$ is the length ratio. For OCNs, $D_{\mathrm{T}}=2$ (Maritan et al., 1996). For natural river networks, data sets show that typically, $1.7<D_{\mathrm{T}}<1.8$. The class of Tokunaga networks predicts values of $D_{\mathrm{T}}$ less than or equal to 2 . The Hack exponent for Tokunaga networks is $\beta_{\mathrm{T}}=1 / D_{\mathrm{T}} \geq 1 / 2$, and the area exponent $\alpha_{\mathrm{T}} \leq 1 / 2$, as observed empirically (Peckham, 1995b). Moreover, for Tokunaga networks, $\alpha_{\mathrm{T}}+\beta_{\mathrm{T}}=1$ (Peckham and Gupta, 1999). A new theory of random self-similar networks (RSN) includes statistical variability, and the Tokunaga is shown as a special case for a subclass of RSN that obey mean selfsimilarity (Veitzer and Gupta, 2000). The RSN theory provides the topologic and geometric foundations on which a $\mathrm{H}-$ $\mathrm{G}$ theory incorporating statistical fluctuations can be developed in the future. RSN theory is not used here.

\subsection{Method: asymptotic self-similarity of type- 1 and type-2}

The scope of the material covered in this subsection is enormous with a long history. We have limited our review to some key concepts that are used in our paper. The reader may consult the well-written books (Barenblatt, 1996, 2003) as required for further explanations.

Dimensional analysis is based on the simple idea that the laws of nature are independent of the arbitrarily chosen basic unit of measurements. As a consequence these laws are invariant under a change of scale. Mathematically this is expressed as a generalized homogeneity that manifests as power laws. The famous Buckingham Pi theorem, or simply $\Pi$ theorem, is a conceptualization of this powerful idea. It enables one to reduce the number of arguments in the functions expressing physical laws, thereby making it simpler to study nature's regularities either experimentally or theoretically. The $\Pi$ theorem says (Barenblatt, 2003, p. 25): "A physical relationship between a dimensional quantity and several dimensional governing parameters can be rewritten as a relationship between a dimensionless parameter and several dimensionless products of the governing parameters. The number of dimensionless products is equal to the total number of governing parameters minus the number of governing parameters with independent dimensions". Let, $a$ be the dependent dimensional quantity, and let $a_{1}, \ldots, a_{k}, b_{1}, \ldots, b_{m}$ represent the $k+m=n$ governing parameters, $k$ of them with independent dimensions. With this notation $a=f\left(a_{1}, \ldots, a_{k}, b_{1}, \ldots, b_{m}\right)$ can be rewritten as $\Pi=\Phi\left(\Pi_{1}, \ldots, \Pi_{m}\right)$, with $\Pi=a a_{1}^{-p} \ldots a_{k}^{-r}$, and the dimensionless products for the governing parameters with independent dimensions are $\Pi_{i}=b_{i} a_{1}^{-p_{i}} \ldots a_{k}^{-r_{i}}$ for $i=1, \ldots, m$. Because of the definitions, the exponents $p_{i}, \ldots, r_{i}$ can be obtained by solving elementary linear equations. A classical example to illustrate the $\Pi$ theorem is the formula for the period $\theta$ of the small oscillations of a pendulum of mass $m$ and length $\ell$ and the gravitational acceleration $g$. The three governing parameters $(m, \ell, g)$ have three independent dimensions ( $\left.\mathrm{M}, \mathrm{L}, \mathrm{LT}^{-2}\right)$. Therefore, the number of dimensionless products of the governing parameters is $n-k=m=3-3=0$, which implies that the dimensionless product involving the period, $\Pi$, is a constant. It can be written as $\theta g^{1 / 2} \ell^{-1 / 2}=$ constant (Barenblatt, 2003, p. 132). The constant cannot be obtained from dimensional analysis. It must be determined either from a theory or from observations. It is $2 \pi$ for the pendulum example.

The majority of the successful examples of the applications of dimensional analysis can be found in many textbooks, which share another important property that is not always emphasized, but which is necessary in our context. For such problems, there is a clear way of separating the important variables from the ones that do not play a significant role in the limit because they are either too small or too large. For instance, the textbook example (Gibbings, 2011, p. 119) of the derivation of Kepler's third law from dimensional analysis needs three non-dimensional numbers, and expresses the ratio of the period of rotation, $T$, in terms of the other two, namely, the ratio of the mass of the planet $m$ to the mass of the Sun $M$ and the ratio of axes of the ellipsis, $a / b$, which can be expressed in terms of the eccentricity, $e^{2}=1-(b / a)^{2}$. Let $G$ denote the constant of gravitation. Then,

$\frac{G M T^{2}}{a^{3}}=f(m / M, e)$.

The crucial observation is that two of the dimensionless numbers are small and therefore do not play a significant role 
in the limit when they go to zero. The consequence is that the limit of the function that expresses the non-dimensional number involving the period of the rotation in terms of the other two goes to a constant. It is well known from the theory that the limit is $4 \pi^{2}$. This is self-similarity characterized by the existence of a non-trivial (different from zero or infinity) limit of the function when some of the other nondimensional products become very small or large. This is called "asymptotic self-similarity of the first kind", or SS1 for short (Barenblatt, 2003, p. 84). SS-1 is applied to our problem in Sects. 6 and 7.

In many other cases, a dimensionless number, despite being too small (or large, if one considers its reciprocal), cannot be ignored in the limit. Mathematically, this corresponds to the case where the limit of a function does not exist, or is zero or infinity. The simplicity of SS-1 that consists in discarding small dimensionless variables and obtaining the scaling exponents from dimensional analysis is lost in this case. In such cases small variables continue to play a role in the problem, and require a generalization of the dimensional analysis. The concept of "asymptotic self-similarity of the second kind", or SS-2 for short, discussed in Barenblatt (1996, chap. 5) is needed. It is known as the "renormalization group theory" in statistical physics. For our purposes, the fluid-mechanical approach is more natural than the statistical physical approach, because it is based on a generalization of the dimensional analysis framework. We follow the fluidmechanical approach in this paper. Barenblatt (1996, p. 172) has explained that these two approaches are equivalent.

A simple example of SS-2 is the determination of the length of a fractal curve, which can be contrasted with a smooth curve (Barenblatt, 2003, p. 132). Let $L_{\eta}$ be the length of a broken line of segment length $\xi$ that approximates the continuous curve between two points that are separated by a distance $\eta . L_{\eta}$ depends on the two dimensional parameters $\eta$ and $\xi$. Dimensional analysis gives $L_{\eta}=\eta f(\eta / \xi)$. For a smooth curve, say a semicircle, as $\xi \rightarrow 0$ the argument $\eta / \xi \rightarrow \infty$ and the function $f$ goes to a limit, namely $\pi / 2$. Whereas for a fractal curve, the limit of $f$ when $\eta / \xi \rightarrow$ $\infty$ is infinity. In fact from fractal geometry we know that $f(\eta / \xi) \simeq(\eta / \xi)^{D-1}$. The anomalous exponent $D>1$ is the fractal dimension that cannot be estimated from dimensional analysis. Barenblatt (1996) gives a recipe for the applications of similarity analysis and SS-2 to obtain the exponents along with many physical examples that include turbulent shear flows, fractals, biological allometry, and groundwater hydrology. We apply SS-2 in Sect. 8.

\section{Data sets}

We use data sets from four drainage basins to test our theory. Three of them are taken from the literature. The fourth one is the experimental Whitewater basin, Kansas, USA that is unpublished. These basins are selected with the criteria of availability of either complete $\mathrm{H}-\mathrm{G}$ data for the entire river network in them or the possibility of carrying out Hortonian analysis for the $\mathrm{H}-\mathrm{G}$ variables in one or more basins. These variables are indexed by the Horton order, $\omega$. We are particularly interested in the power-law representation of the $\mathrm{H}-$ $\mathrm{G}$ variables as functions of discharge that is widely used in the literature for analyzing data (see Sect. 6.1 for a theoretical derivation). The power-law representation and the corresponding scaling exponents are defined below. Our notation is the same as in Leopold et al. (1964, p. 244).

$U_{\omega} \propto Q_{\omega}^{m}, \quad W_{\omega} \propto Q_{\omega}^{b}, \quad D_{\omega} \propto Q_{\omega}^{f}$,

$S_{\omega} \propto Q_{\omega}^{z}$ and $n_{\omega}^{\prime} \propto Q_{\omega}^{y}$.

The first basin is the classic Brandywine creek, PA in the US as given in Leopold et al. (1964, Table 7.5, p. 244), where the $\mathrm{H}-\mathrm{G}$ exponents are also given. It has humid subtropical climate with cool to cold winters, hot, humid summers, and generous precipitation throughout the year, approx. $1100 \mathrm{~mm}$ year $^{-1}$. Köppen climate classification lists it as type Cfa (Köppen, 1884). It has a drainage area of $777 \mathrm{~km}^{2}$ at the mouth. Average discharge is $12 \mathrm{~m}^{3} \mathrm{~s}^{-1}$. The observed values of the $\mathrm{H}-\mathrm{G}$ exponents for the Brandywine creek (Leopold et al., 1964, Table 7.5, p. 244) are $b=0.42$, $f=0.45, m=0.05, z=-1.07$ and $y=-0.28$.

Two comprehensive field measurement programs were conducted in NZ. The largest part of NZ has a pleasant sea climate with mild winters and warm summers. Köppen climate classification lists it as type Cf. The second field study was conducted in the Taieri River basin (Ibbitt et al., 1998). Figure 1 illustrates the measurement sites in the channel network of the Taieri River basin. It has an estimated mean annual precipitation of $1400 \mathrm{~mm}$. The basin area is $158 \mathrm{~km}^{2}$. Mean discharge, as measured over the discontinuous 14-year period 1983-1996, is $4.90 \mathrm{~m}^{3} \mathrm{~s}^{-1}$, representing an average runoff rate of $980 \mathrm{~mm}_{\text {year }}{ }^{-1}$ from the basin. The field values of the $\mathrm{H}-\mathrm{G}$ exponents for the Taieri River basin (Ibbitt et al., 1998) are $b=0.517, z=-0.315, m=0.238$ and $f=0.247$.

The third field study was conducted in the $121 \mathrm{~km}^{2}$ Ashley River basin (McKerchar et al., 1998). Annual precipitation increases in a northwesterly direction across the basin from 1200 to about $2000 \mathrm{~mm}$ year $^{-1}$. Mean discharge, as measured by the stream gauge over the 20 -year period $1977-$ 1996 , is $3.99 \mathrm{~m}^{3} \mathrm{~s}^{-1}$, representing an average runoff rate of $1040 \mathrm{~mm} \mathrm{year}^{-1}$ from the basin. The observed $\mathrm{H}-\mathrm{G}$ scaling exponents for the Ashley River basin (McKerchar et al., 1998) are $b=0.44, z=-0.317, m=0.318$ and $f=0.242$. The Horton analysis for this basin is briefly described in Sect. 8.4. Both the data sets from NZ are unique, and offer the potential to conduct Horton analysis for the $\mathrm{H}-\mathrm{G}$ variables that Mantilla (2014) is conducting. Some of his results are presented in Sect. 8.4.

The last data set comes from the $1100 \mathrm{~km}^{2}$ Whitewater, Kansas in the US. According to the Köppen climate classification, the climate of this part of Kansas can be characterized 


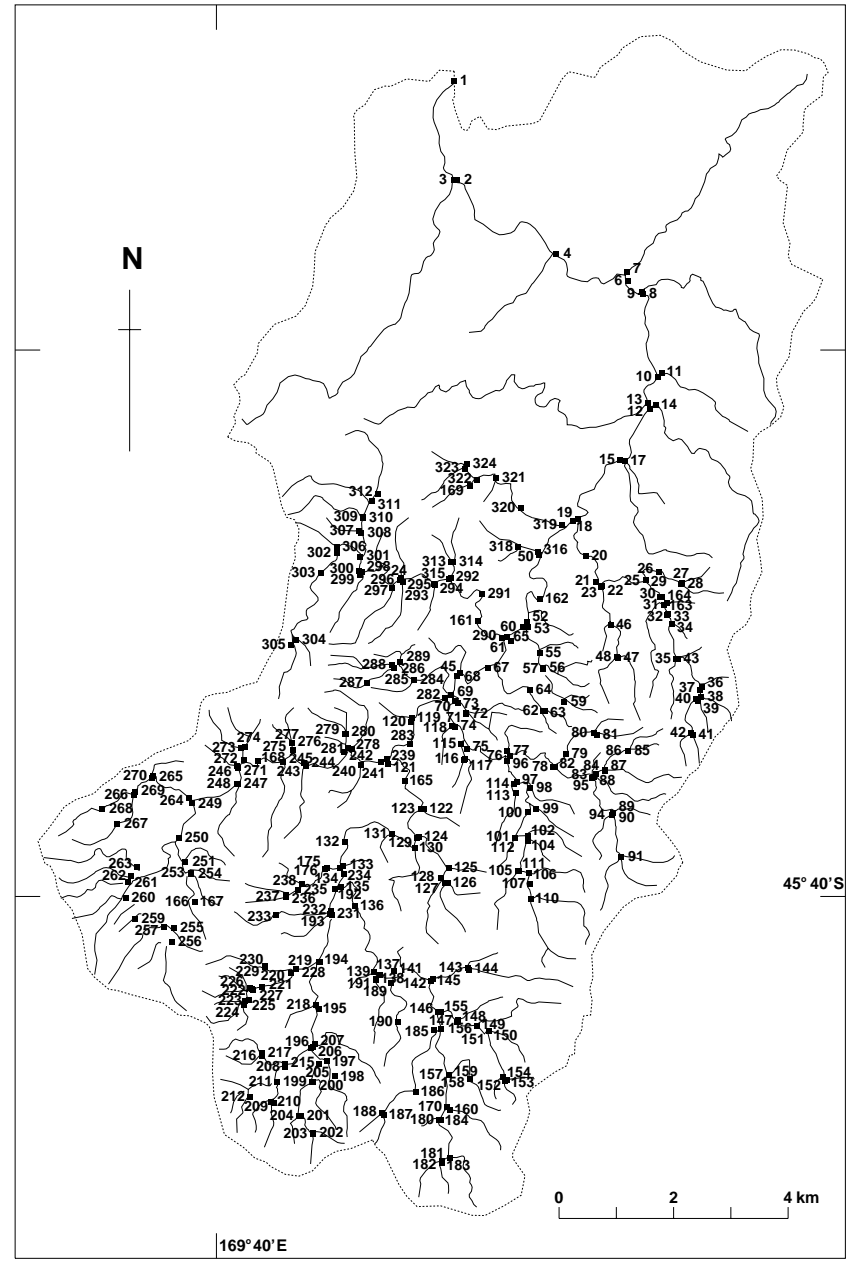

Figure 1. Reproduction of the original figure of Ibbitt et al. (1998, Fig. 1), showing the river network of the Taieri basin in New Zealand along with the measurement sites in the network.

as a humid continental climate, with cool to cold winters and hot, often humid summers. Most of the precipitation falls in summer and spring. Average precipitation is of the order of $840 \mathrm{~mm}$ year $^{-1}$. Snowmelt runoff is negligible, soil is composed of fine-grained sediment, and the land use is primarily agricultural and cattle ranching. Whitewater basin was instrumented to conduct an interdisciplinary field and theoretical hydrology program, called Hydro-Kansas (2002-2012). Kean and Smith (2005) tested a theoretical fluid-mechanical model to estimate stream flows. The model was used to estimate streamflows at multiple spatial locations in streams of different Strahler order in the Whitewater basin (Clayton and Kean, 2010). Mantilla (2014) participated in the H-K program and took field measurements of some of the $\mathrm{H}-\mathrm{G}$ variables, especially width and depth. He has kindly shared the necessary data for our study.

An important issue in estimating field values of the $\mathrm{H}-$ $\mathrm{G}$ exponents is the measurement errors that are inherent to measuring the $\mathrm{H}-\mathrm{G}$ variables, which affect their scaling ex- ponents. For example, Ibbitt et al. (1998) and McKerchar et al. (1998) have given standard errors for each of the H$\mathrm{G}$ exponents that are listed in Table 1. We use these fieldestimated values in testing theoretical predictions against their observed values from three basins in Sects. 6 and 8.3. Theoretical relations between the Horton laws for the $\mathrm{H}-\mathrm{G}$ variables and the corresponding scaling exponents are briefly considered in Sect. 8.4.

\section{Dimensionless river-basin numbers}

The fundamental physical parameters governing the $\mathrm{H}-\mathrm{G}$ in drainage networks are defined at the bottom of a complete Strahler stream of order $\omega \geq 1$ as follows. $Q_{\omega}$ is the river discharge rate $\left(\mathrm{L}^{3} \mathrm{~T}^{-1}\right), A_{\omega}$ is the cumulative drainage area, and $D_{\omega}, U_{\omega}$, and $W_{\omega}$ are channel depth, velocity, and width, respectively. $H_{\omega}$ is the elevation drop that is defined as the elevation difference between the beginning and end junctions of a complete Strahler stream. $L_{\omega}$ denotes the corresponding stream length. Slope is defined as $S_{\omega}=H_{\omega} / L_{\omega}$. Kinematic viscosity is $\nu$, water density is $\rho$, and the gravitational acceleration is $g . R$ is the mean runoff rate per unit area from the hillslopes along a channel network, and has dimension $\mathrm{L} \mathrm{T}^{-1}$. The spatial uniformity of $R$ implies that the river basin is assumed to be homogeneous with respect to mean runoff generation. This assumption can be relaxed, but we want to keep this presentation simple.

From the set of twelve variables listed above, only nine are independent, because three variables are dependent: $Q_{\omega}=$ $U_{\omega} W_{\omega} D_{\omega}, S_{\omega}=H_{\omega} / L_{\omega}$, and $L_{\omega}=A_{\omega}^{1 / D_{\mathrm{T}}}$. These nine independent variables include three basic dimensions, length $(L)$, time $(T)$ and mass $(M)$. The Buckingham Pi theorem explained in Sect. 2.2 gives that the number of independent dimensionless numbers is 6 , but it does not give what they are. They can be specified either using formal dimensional analysis or physical arguments. We adopt the later approach because it is physically insightful. Some of these dimensionless numbers were considered in Peckham (1995b).

The first dimensionless number is given by

$\Pi_{1}(\omega)=\frac{Q_{\omega}}{R A_{\omega}}$.

The discharge rate $Q_{\omega}$ is taken to be a linear function of drainage area given by $Q_{\omega}=R A_{\omega}$, which is observed in many humid climates for low and mean flows. Leopold et al. (1964) used mean flow in their $\mathrm{H}-\mathrm{G}$ investigations. Low flow has been used in recent field $\mathrm{H}-\mathrm{G}$ observations from NZ that are explained in Sect. 3 and analyzed in Sect. 8.

The second dimensionless number is

$\Pi_{2}(\omega)=\frac{R \sqrt{A_{\omega}}}{D_{\omega} U_{\omega}}$.

It is suggested by mass conservation involving the ratio of runoff per unit width of drainage basin in the numerator, and discharge per unit channel width in the denominator. 
Table 1. Summary of observed and predicted H-G scaling exponents. The sources of the data are Ibbitt et al. (1998) for the Taieri River basin in New Zealand, McKerchar et al. (1998) for the Ashley River basin in New Zealand, and Leopold et al. (1964, Table 7.5, p. 244) for the Brandywine creek, PA in the United States.

\begin{tabular}{|c|c|c|c|c|}
\hline \multirow[b]{2}{*}{ Variable } & \multirow[b]{2}{*}{ Exponent } & \multicolumn{3}{|c|}{ Basin } \\
\hline & & Taieri & Asheley & Brandywine \\
\hline \multicolumn{5}{|c|}{ Observed } \\
\hline$U \propto Q^{m}$ & $m$ & $0.238 \pm 0.023$ & $0.318 \pm 0.018$ & 0.050 \\
\hline$W \propto Q^{b}$ & $b$ & $0.517 \pm 0.016$ & $0.440 \pm 0.016$ & 0.420 \\
\hline$D \propto Q^{f}$ & $f$ & $0.247 \pm 0.016$ & $0.242 \pm 0.014$ & 0.450 \\
\hline$S \propto Q^{z}$ & $z$ & $-0.315 \pm 0.026$ & $-0.317 \pm 0.020$ & -1.070 \\
\hline$n^{\prime} \propto Q^{y}$ & $y$ & $-0.231 \pm 0.028$ & $-0.315 \pm 0.023$ & -0.280 \\
\hline$U D / v \propto Q^{m+f}$ & $m+f$ & $0.485 \pm 0.028$ & $0.560 \pm 0.023$ & 0.500 \\
\hline \multicolumn{5}{|c|}{ Estimated using $D_{\mathrm{T}}=7 / 4, f$ and $z$} \\
\hline & $\alpha$ & 0.208 & 0.175 & 0.441 \\
\hline & $\beta$ & -0.822 & -0.864 & 0.327 \\
\hline \multicolumn{5}{|c|}{ Predicted using Eqs. (28), (45) and (53). } \\
\hline$U D / v \propto Q^{m+f}$ & $m+f$ & 0.500 & 0.500 & 0.500 \\
\hline$W \propto Q^{b}$ & $b$ & 0.500 & 0.500 & 0.500 \\
\hline$n^{\prime} \propto \widetilde{Q}^{y}$ & $y$ & $-0.246 \pm 0.030$ & $-0.255 \pm 0.025$ & -0.285 \\
\hline
\end{tabular}

The relation between gravitational and inertia forces in river networks suggests the third dimensionless number $\Pi_{3}(\omega)$. Specifically, we define the "basin Froude number" as

$\Pi_{3}(\omega)=\frac{U_{\omega}}{\sqrt{g H_{\omega}}}=\frac{U_{\omega}}{\sqrt{g S_{\omega} L_{\omega}}}$,

where the channel slope

$\Pi_{4}(\omega)=S_{\omega}=H_{\omega} / L_{\omega}$

is the fourth dimensionless number. The drop $H_{\omega}$ defines the length scale governing the gravitational force. It should be differentiated from a channel Froude number in open channel hydraulics where flow depth defines the length scale.

The fifth dimensionless number is given by the Reynolds number. Leopold et al. (1964, p. 158) have discussed its significance in the context of laminar and turbulent flows. In natural streams, the flow is largely turbulent.

$\Pi_{5}(\omega)=\frac{U_{\omega} D_{\omega}}{v}$.

The sixth dimensionless number incorporates the factors controlling flow velocity. The total frictional force along the channel boundary is $\tau_{\omega}\left(2 D_{\omega}+W_{\omega}\right) L_{\omega} \approx \tau_{\omega} W_{\omega} L_{\omega}$, where $\tau_{\omega}$ is the shear stress per unit area. It is proportional to the square of the mean velocity for turbulent flows if the boundary does not change with variations in flow (Leopold et al., 1964, p. 157). The gravitational force due to the mass of water along the channel length $L_{\omega}$ is given by $\rho g W_{\omega} D_{\omega} L_{\omega} S_{\omega}$.
The dimensionless ratio of these two forces gives

$\Pi_{6}(\omega)=\frac{U_{\omega}^{2}}{g D_{\omega} S_{\omega}}$.

The term $\sqrt{g D_{\omega} S_{\omega}}$ is known as the shear velocity. $\Pi_{6}$ is proportional to the Darcy-Weisbach resistance coefficient. Leopold et al. (1964, Fig. 6.5) illustrated that for the Brandywine Creek, PA, $1 / \sqrt{\Pi_{6}}$ is linearly related to the logarithm of relative roughness defined by the ratio of flow depth to the height of roughness elements.

\section{Mass conservation in self-similar Tokunaga networks}

The discharge $Q_{\omega}$ is computed using a mass conservation equation for a network indexed by the Strahler or$\operatorname{der} \omega \geq 1$. We show that a mass conservation equation for a channel network indexed by link magnitudes (Gupta and Waymire, 1998) is a special case of it. We further assume that our channel network is self-similar Tokunaga with uniform link lengths $l$. No statistical fluctuations in the topology of channel network are considered in developing the $\mathrm{H}-\mathrm{G}$ theory as mentioned in Sect. 2.1.

Let $\bar{S}_{\omega}(t)$ denote the storage in a Strahler stream of order $\omega \geq 1$ defined by

$\bar{S}_{\omega}(t)=W_{\omega}(t) D_{\omega}(t) L_{\omega}$.

The dependence of storage on time $t$ comes from temporal variations of streamflows in the network, which result in width and depth varying with time. 
The total number of junctions denoted by $C_{\omega}$ is the same as the total number of links in a complete Strahler stream of or$\operatorname{der} \omega$. Let $t_{i}, i=1,2,3, \ldots, C_{\omega}$ be a sequence of travel times for water to reach the bottom of a complete Strahler stream from successive junctions enumerated from the bottom. This means that $t_{1}$ represents the travel time from the first junction from the bottom, $t_{2}$ from the second junction and so on. For example, if water flows with a uniform velocity $u$, then $t_{i}=i l / u$, because all the links are assumed to have the same length $l$.

Let $R_{i}(t), i=1,2,3, \ldots, C_{\omega}$ denote the volumetric runoff rate from the $i$ th hill along a complete Strahler stream of order $\omega$. Let $Q_{k_{i}}, i=1,2, \ldots, C_{\omega}$ denote the discharge from the side tributary at the $i$ th junction from the bottom. Here the subscripts $k_{1}, k_{2}, \ldots$ denote the Strahler orders of the side tributaries coming into the junctions counted from the bottom of a stream. Let $Q_{\omega-1}^{1}(t)$ and $Q_{\omega-1}^{2}(t)$ denote the discharges in the two tributaries at the top of the stream. Each of them is of order $\omega-1$ by definition of the Strahler ordering given in Sect. 2.

Considering a Strahler stream as a finite control volume, the mass conservation equation can be written as

$$
\begin{aligned}
\frac{\mathrm{d} \bar{S}_{\omega}(t)}{\mathrm{d} t} & +Q_{\omega}(t)=Q_{\omega-1}^{1}\left(t-t_{\omega-1}\right)+Q_{\omega-1}^{2}\left(t-t_{\omega-1}\right) \\
& +\sum_{i=1}^{C_{\omega}} Q_{k_{i}}\left(t-t_{i}\right)+2 \sum_{i=1}^{C_{\omega}} R_{i}(t) .
\end{aligned}
$$

For $\omega=1$, Eq. (15) reduces to the link magnitude-based mass conservation equation in Gupta and Waymire (1998) that is easy to check.

As a first step, we have chosen to focus solely on the spatial analysis in the context of $\mathrm{H}-\mathrm{G}$. In particular, we seek a spatial solution of Eq. (15) by ignoring the time dependence of $Q_{\omega}(t)$, and denoting it as $Q_{\omega}(t)=Q_{\omega}$. This is tantamount to assuming that $\mathrm{d} \bar{S}_{\omega}(t) / \mathrm{d} t=0, R_{i}(t)=0, \forall i, t>0$, and the travel times $t_{i}=0, \forall i$. Physically, these sets of assumptions can be interpreted to mean that $R$, is applied uniformly throughout the network at time $t=0$. Moreover, water is assumed to travel in a very short time throughout the network so that travel times are ignored.

In a recent paper on a space-time theory of low flows for river networks, travel times were ignored throughout the basin compared to the subsurface response time for hillslopes, and $R$ was computed from hillslope processes under idealized conditions. The theoretical results thus obtained compared well with observations (Furey and Gupta, 2000). Similarly, in the present context, the idealized assumptions made above are necessary for making progress on this complex problem.

In view of the above assumptions, Eq. (15) simplifies to

$Q_{\omega}=Q_{\omega-1}^{1}+Q_{\omega-1}^{2}+\sum_{i=1}^{C_{\omega}} Q_{k_{i}}$
The key problem is to compute a solution for $Q_{\omega}$. In view of the definition of self-similarity given in Sect. 2.1, Eq. (16) reduces to

$Q_{\omega}=2 Q_{\omega-1}+\sum_{k=1}^{\omega-1} T_{k} Q_{\omega-k}$

where $T_{k}=T_{\omega, \omega-k}, k=1,2, \ldots, \omega-1$ denotes the number of side tributaries of order $\omega-k$ joining a stream of order $\omega$. Equation (17) has been solved rigorously under the assumption that $T_{k}$ 's obey Tokunaga self-similarity. The solution is given by Eq. (4). Because the recursion equation (Eq. 17) for $Q_{\omega}$ is the same as the one for $A_{\omega}$, we assert from the arguments given in Sect. 2.1 that

$$
\begin{aligned}
R_{Q}=\lim _{\Omega-\omega \rightarrow \infty} \frac{Q_{\omega+1}}{Q_{\omega}}=R_{B} & =\lim _{\Omega-\omega \rightarrow \infty} \frac{N_{\omega}}{N_{\omega+1}} \\
& =R_{A}=\lim _{\Omega-\omega \rightarrow \infty} \frac{A_{\omega+1}}{A_{\omega}}
\end{aligned}
$$

and

$R_{Q}=R_{B}=R_{A}=\frac{(2+a+c)+\sqrt{(2+a+c)^{2}-8 c}}{2}$.

Equation (18) implies that $Q_{\omega}$ equals $R A_{\omega}$, which is used in defining the first dimensionless number in Sect. 4. It follows from the definitions of Horton ratios, and from the equality $R_{Q}=R_{B}$, that

$Q_{\omega+1} N_{\omega+1}=Q_{\omega} N_{\omega}$, as $\Omega-\omega \rightarrow \infty$.

This is a foundational result governing mass conservation in self-similar Tokunaga networks. Even though, Eq. (19) is valid in the limit of large network order, the result holds for small values of $\omega$ as explained in Sect. 2.1. It should be noted that if one substitutes $A_{\omega}$ for $Q_{\omega}$ in Eq. (19), then it loses its physical interpretation. The reason is that $Q_{\omega}$ is a dynamic variable but $A_{\omega}$ is a fixed geometrical variable. Moreover, a power law relation between these two variables plays a fundamental role in the $\mathrm{H}-\mathrm{G}$ investigations as explained above in Sect. 2 and later in Sect. 8.3.

West et al. (1997) used the mass conservation equation for perfect branching biological networks in which no side tributaries are present, and in which each parent branch bifurcates into two branches. In that case, it is simple to write down Eq. (19) as a special case of mass conservation without involving any limit. West et al. (1997) used it to obtain some remarkable results governing allometry in biological networks.

We apply Eq. (19) to extend the geometric and topological Horton laws in self-similar Tokunaga networks to include the $\mathrm{H}-\mathrm{G}$ variables. Figure 2 shows a Horton law for channel widths in a drainage network that was mentioned along with other $\mathrm{H}-\mathrm{G}$ variables in Sect. 2. As mentioned there, the key equation providing this link is a power-law relation between discharge and drainage area, and a Horton law for drainage 
areas (Leopold and Miller, 1956, pp. 19-20). In our context, Eq. (18) showing that $R_{Q}=R_{A}$ represents both these features for Tokunaga networks.

\section{Derivation of Horton laws and prediction of the width and Reynolds number exponents using SS-1}

\subsection{Horton laws for channel width, depth and velocity}

It follows from the definition of $\Pi_{1}$ in Eq. (8), and from the identity in Eq. (18) that $R_{Q}=R_{A}$,

$\lim _{\Omega-\omega \rightarrow \infty} \frac{\Pi_{1}(\omega+1)}{\Pi_{1}(\omega)}=R_{\Pi_{1}}=1, \quad \omega=1,2, \ldots \ll \Omega$.

This important result comes from the self-similarity of Tokunaga networks and the assumption of spatial homogeneity of runoff $R$. It probably is a valid assumption for widely varying climatic regions and a broad range of spatial scales. It is tested in Sect. 8.4 for the Ashley River basin from NZ that is described in Sect. 3.

The five $\mathrm{H}-\mathrm{G}$ variables, $U, W, D, S$ and $n^{\prime}$ considered in this paper vary as discharge $Q$ varies, which is the sixth $\mathrm{H}-\mathrm{G}$ variable. Therefore, we assume that all the $\mathrm{H}-\mathrm{G}$ variables are homogeneous functions of $Q$. This is the simplest mathematical assumption because it means that the functions do not depend on any other parameter except $Q$. It is widely used in the $\mathrm{H}-\mathrm{G}$ literature reviewed in Sect. 2. We can write it as $U=f_{1}(Q), W=f_{2}(Q)$, etc. To determine the form of these functions, we need a functional equation that applies to our physical context. Peckham (1995b, p. 53) has reviewed four functional equations with solutions known as Cauchy equations that repeatedly come up in similarity type investigations. Of these four, the most pertinent in our context is the equation based on self-similarity. Consider $U=f_{1}(Q)$. Self-similarity can be represented by $f_{1}\left(Q_{1} \cdot Q_{2}\right)=f_{1}\left(Q_{1}\right) f_{2}\left(Q_{2}\right)$ (Gupta and Waymire, 1998, pp. 102-103), whose solution is a power law. Therefore,

$U=f_{1}(Q) \propto Q^{m}$.

The above argument applies to all the functions. Therefore, the $\mathrm{H}-\mathrm{G}$ variables can be written as power law functions of discharge, . This functional form has been widely used in $\mathrm{H}-\mathrm{G}$ investigations (Leopold and Miller, 1956), and was introduced in Eq. (7).

Equation (18) shows that the ratio, $Q_{\omega+1} / Q_{\omega}$, converges to $R_{Q}$, and thereby obeys a Horton law. To extend the Horton laws to other $\mathrm{H}-\mathrm{G}$ variables, let us consider velocity

$\lim _{\Omega-\omega \rightarrow \infty} \frac{U_{\omega+1}}{U_{\omega}}=\frac{Q_{\omega+1}^{m}}{Q_{\omega}^{m}}=R_{Q}^{m}=R_{U}$,

which follows from the fact that the ratios are positive and monotonic in $\omega$ (Rudin, 1976, p. 44). Similarly, $R_{W}=R_{Q}^{b}$ and $R_{D}=R_{Q}^{f}$. By definition, $Q_{\omega}=U_{\omega} W_{\omega} D_{\omega}$. Therefore,

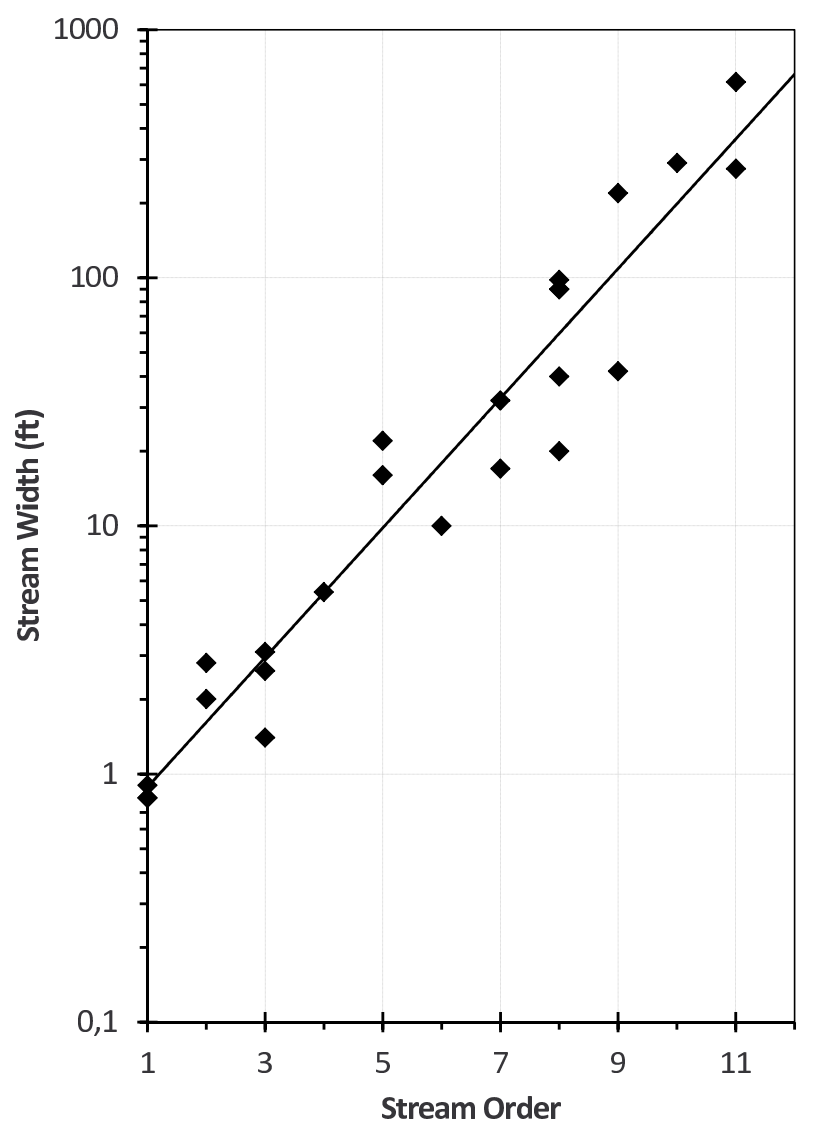

Figure 2. Reproduction of the original figure of Leopold and Miller (1956, Fig. 19, p. 23), showing the relation of stream width to stream order in arroyos.

the Horton ratios for velocity, width and depth can be written as

$R_{U}=R_{Q}^{m}, \quad R_{W}=R_{Q}^{b}, \quad R_{D}=R_{Q}^{f}, \quad m+b+f=1$.

The derivation of Eq. (22) required that (i) Horton laws for channel widths, depths and velocities hold in Tokunaga self-similar networks, (ii) runoff generation $R$ is spatially homogeneous, and that (iii) channel width, depth and velocity depend monotonically on Strahler order $\omega$.

\subsection{Prediction of the width exponent and Reynolds number exponent}

We will now use the above results to show that the Horton laws for the topologic and geometric variables explained in Sect. 2.1 extend to channel widths. Our arguments are based on dimensional analysis, as explained in Sect. 2.2. Consider the dimensionless number $\Pi_{2}(\omega)$ defined by Eq. (9), and the ratio given by

$$
R_{\Pi_{2}}(\omega)=\frac{\sqrt{A_{\omega+1}}}{\sqrt{A_{\omega}}} \cdot \frac{D_{\omega} U_{\omega}}{U_{\omega+1} D_{\omega+1}} .
$$


Substituting $D_{\omega} U_{\omega}=Q_{\omega} / W_{\omega}$ in the above expression gives

$R_{\Pi_{2}}(\omega)=\frac{\sqrt{A_{\omega+1}}}{\sqrt{A_{\omega}}} \cdot \frac{Q_{\omega} W_{\omega+1}}{Q_{\omega+1} W_{\omega}}$.

We have already shown that the right-hand side converges to a constant in Sect. 6.1. It follows that the left-hand side of Eq. (24) also converges to a constant. Stated mathematically,

$$
\begin{aligned}
\lim _{\Omega-\omega \rightarrow \infty} & \frac{\sqrt{A_{\omega+1}}}{\sqrt{A_{\omega}}} \frac{Q_{\omega} W_{\omega+1}}{Q_{\omega+1} W_{\omega}}=\frac{R_{A}^{1 / 2} R_{W}}{R_{Q}} \\
& =\lim _{\Omega-\omega \rightarrow \infty} R_{\Pi_{2}}(\omega)=R_{\Pi_{2}} .
\end{aligned}
$$

The asymptotic constancy of the ratio $R_{\Pi_{2}}(\omega)$ of the dimensionless number $\Pi_{2}$ across different Strahler orders holds in Tokunaga networks. Since $R_{Q}=R_{A}$, Eqs. (25) and (22) can be combined to obtain

$R_{W}=R_{\Pi_{2}} R_{Q}^{1 / 2}=R_{Q}^{b}$.

Therefore, $R_{\Pi_{2}}=1$, and the channel width $\mathrm{H}-\mathrm{G}$ exponent is

$R_{W}=R_{Q}^{1 / 2}, \quad b=1 / 2$.

It follows directly from Eqs. (12), (22) and (27) that a Horton law for the Reynolds number can be written as

$R_{\Pi_{5}}=R_{U} R_{D}=R_{Q}^{m+f}=R_{Q}^{1 / 2}$.

We now test these predictions against data from three field studies described in Sect. 3 and Table 1. The first test of the theory is for the Brandywine creek (Leopold et al., 1964, Table 7.5, p. 244). The empirical values of width, depth and velocity do not satisfy $b+f+m=1$, instead they add to 0.92 . Assuming that depth and velocity exponents are correct as per our discussion in Sect. 8.3, $b=1-f-m=1 / 2$ agrees with our theoretical prediction. Likewise, $f+m=1 / 2$ also agrees with our predictions.

The second test of the theory is in Taieri River basin (Ibbitt et al., 1998). The empirical width exponent interval is $(0.501,0.533)$. The predicted value $b=1 / 2$ is very close to the empirically estimated lower bound. For the computation of the error in $m+f$ we assume independence and use the well-known formula that the variance of a sum is the sum of variances. It is used whenever there is a linear function of the exponents. Using the formula, the empirical depth plus velocity exponent interval is $(0.457,0.513)$. The predicted value $m+f=1 / 2$ lies in this range. Both these predictions support our theory in the Taieri basin.

The third test is in the Ashley River basin (McKerchar et al., 1998). The empirical width exponent interval is $(0.424$, $0.456)$. The predicted value $b=1 / 2$ lies outside this range. Similarly, the empirical depth plus velocity exponent interval is $(0.537,0.583)$. The predicted value $m+f=1 / 2$ lies outside the range. Clearly there is a discrepancy between the observed and the predicted values.
Comparing the field-measured values of the $\mathrm{H}-\mathrm{G}$ exponents in the Taieri basin and the Ashley basin, which have similar scales and climates, $f$ and $z$ are comparable as one expects, but not $b$ and $m$. A physical reason for this discrepancy is diagnosed in Sect. 8.4.

\section{Predictions of Horton laws and the H-G exponents assuming SS-1, and comparison with OCN model exponents}

In the following developments, we tentatively assume that SS-1 applies to slope, $S_{\omega}$. Similar to the physical assumptions made before for channel width, depth and velocity, we assume that slope depends monotonically on Strahler order. Therefore, the Horton ratio for slope converges to $R_{S}$ following a similar reasoning as given in Eq. (21). From that assumption we predict the Horton laws for width, depth, velocity and slope and test our predictions of their exponents against the optimal channel network (OCN) model of Rodríguez-Iturbe et al. (1992). We show that the four predicted scaling exponents based on this tentative assumption agree with the OCN model. However, our theory differs from it in a fundamental manner because we predict Horton laws for these variables, but the OCN model does not address this issue. Moreover, our analysis uses self-similarity, but OCN uses "optimality". SS- 1 in the $\mathrm{H}-\mathrm{G}$ context is not the correct assumption as explained in the next section. It is being made here only to compare the predictions of the $\mathrm{H}-\mathrm{G}$ exponents from our theory with the OCN model.

Define the Horton ratio for the basin Froude number from Eq. (10). Following similar arguments as given in Eq. (21), and given about the length ratio in Sect. 2.1, we assert the convergence of the basin Froude number because the Horton ratio of each term in it converges.

$\lim _{\Omega-\omega \rightarrow \infty} \frac{\Pi_{3}(\omega+1)}{\Pi_{3}(\omega)}=R_{\Pi_{3}}=\frac{R_{U}}{\sqrt{R_{L} R_{S}}}$.

Using the notation in Eq. (7), and applying Eq. (21) to slope $S$, it follows that $R_{S}=R_{Q}^{z}$. In view of Eq. (18) showing that $R_{Q}=R_{A}$, it follows from the results in Sect. 2.1 that $R_{L}=R_{A}^{1 / D_{\mathrm{T}}}=R_{Q}^{1 / D_{\mathrm{T}}}$. Assuming that the OCN model is space filling as discussed in Sect. 2.1, it follows that $D_{\mathrm{T}}=2$. Substituting $R_{U}=R_{Q}^{m}$ from Eq. (21) into Eq. (29) gives

$R_{U}=R_{Q}^{m}=R_{\Pi_{3}} R_{Q}^{1 / 4} R_{Q}^{z / 2}$.

Equation (30) predicts that $R_{\Pi_{3}}=1$, and

$m=\frac{1}{2}(z+1 / 2)$.

Similarly, consider the Horton ratio for the dimensionless number proportional to the Darcy-Weisbach resistance coefficient given by Eq. (13), and take the limit. We have 
demonstrated the convergence of each term in it. Therefore,

$\lim _{\Omega-\omega \rightarrow \infty} \frac{\Pi_{6}(\omega+1)}{\Pi_{6}(\omega)}=R_{\Pi_{6}}=\frac{R_{U}^{2}}{R_{D} R_{S}}$.

We get an expression for the depth exponent by rewriting Eq. (32) as

$$
R_{D}=R_{Q}^{f}=\frac{R_{U}^{2}}{R_{\Pi_{6}} R_{S}}=\frac{R_{Q}^{2 m}}{R_{\Pi_{6}} R_{Q}^{z}} .
$$

It predicts $R_{\Pi_{6}}=1$, and

$f=2 m-z$.

Solving Eqs. (31) and (34) gives $f=1 / 2, m=0$, and $z=-1 / 2$, which also satisfy the constraint $m+f=1 / 2$. Our predictions may be summarized as follows: (1) Horton laws hold for the $\mathrm{H}-\mathrm{G}$ variables in self-similar Tokunaga networks, and (2) the $\mathrm{H}-\mathrm{G}$ exponents are $b=1 / 2, m=0$, $f=1 / 2$, and $z=-1 / 2$. Our second prediction agrees with the OCN model of Rodríguez-Iturbe et al. (1992). We have already mentioned that the OCN model does not consider Horton laws for the $\mathrm{H}-\mathrm{G}$ variables. That the exponents match is not a surprise because our theory is built on dimensional analysis. In conclusion, we state that our theory is fundamentally different from the OCN model.

To test the OCN predictions, we used the data sets reported in Sect. 3 and Table 1. Except for the Brandywine and Taieri basins that support $b=1 / 2$, other measured $\mathrm{H}-\mathrm{G}$ exponents do not agree with theoretical predictions. The deviations are substantial, suggesting that $\mathrm{H}-\mathrm{G}$ in networks does not obey SS-1 for $D, U$ and $S$. We address this foundational issue in the next section.

\section{Application of SS-2 to predict Horton laws for the $H-G$ variables and the exponent of the Manning friction}

Slope appears in dimensionless numbers given by Eqs. (10), (11) and (13). The stream drop in Eq. (11) is bounded but stream length increases with order. Therefore, slope $S_{\omega} \rightarrow 0$ as $\Omega-\omega \rightarrow \infty$, but slope cannot be eliminated from the asymptotic limit. Therefore, SS-1 is not applicable to our problem. A generalization of the dimensional analysis is required to develop the $\mathrm{H}-\mathrm{G}$ theory as explained in Sect. 2.2. It is the focus of this section.

\subsection{Horton laws and theoretical expressions for the H-G exponents}

We define two "renormalized dimensionless numbers" in which slope appears. Equations (10) and (13) modify to

$$
\begin{aligned}
& \Pi_{3}^{*}(\omega)=\frac{U_{\omega}}{\sqrt{g L_{\omega} S_{\omega}^{\alpha}}}, \\
& \Pi_{6}^{*}(\omega)=\frac{U_{\omega}^{2}}{g D_{\omega} S_{\omega}^{\beta}} .
\end{aligned}
$$

Here, $\alpha$ and $\beta$ are "anomalous scaling exponents" that cannot be predicted from dimensional analysis. In principle they can be predicted from physical arguments involving sediment transport. This is a task for future research as explained in Sect. 9.

Following similar arguments as given in Sect. 6, and assuming that slope is monotonic in order as in Sect. 7, Eq. (21) applies to slope. Therefore, we assert the convergence of the ratios of the renormalized dimensionless numbers,

$\lim _{\Omega-\omega \rightarrow \infty} \frac{\Pi_{3}^{*}(\omega+1)}{\Pi_{3}^{*}(\omega)}=R_{\Pi_{3}^{*}}=\frac{R_{U}}{\sqrt{R_{L} R_{S}^{\alpha}}}$.

and,

$\lim _{\Omega-\omega \rightarrow \infty} \frac{\Pi_{6}^{*}(\omega+1)}{\Pi_{6}^{*}(\omega)}=R_{\Pi_{6}^{*}}=\frac{R_{U}^{2}}{R_{D} R_{S}^{\beta}}$.

We have explained in Sect. 7 that $R_{L}=R_{A}^{1 / D_{\mathrm{T}}}=R_{Q}^{1 / D_{\mathrm{T}}}$ and $R_{S}=R_{A}^{z}=R_{Q}^{z}$. From Sect. 6, $R_{U}=R_{Q}^{m}$. Therefore, Eq. (37) gives

$R_{U}=R_{Q}^{m}=R_{\Pi_{3}}^{*} R_{Q}^{1 / 2 D_{\mathrm{T}}} R_{Q}^{z \alpha / 2}$.

Equation (39) predicts that $R_{\Pi_{3}}^{*}=1$, and

$m=\frac{1}{2}\left(z \alpha+1 / D_{\mathrm{T}}\right)$.

Since $m+f=1 / 2$, an expression for the depth exponent follows directly from Eq. (40),

$f=\frac{1}{2}\left(1-z \alpha-1 / D_{\mathrm{T}}\right)$.

We get a second expression for the depth exponent by rewriting Eq. (38) as

$R_{D}=R_{Q}^{f}=\frac{R_{U}^{2}}{R_{\Pi_{6}^{*}} R_{S}^{\beta}}=\frac{R_{Q}^{2 m}}{R_{\Pi_{6}}^{*} R_{Q}^{z \beta}}$.

It predicts, $R_{\Pi_{6}^{*}}=1$, and, in view of Eq. (40),

$f=2 m-z \beta=z(\alpha-\beta)+1 / D_{\mathrm{T}}$. 
Equating the expressions for $f$ from Eqs. (43) and (41), we obtain an expression for the slope scaling exponent as

$z(3 \alpha-2 \beta)=1-3 / D_{\mathrm{T}}$.

Equations (40) and (44) together generalize the H-G theory for a channel network based on an application of SS-2.

To summarize, given the topological fractal dimension $D_{\mathrm{T}}$ and the prediction of the width exponent $b=1 / 2$ by Eq. (28), we have two equations, (40) and (44), which give theoretical expressions for $\mathrm{H}-\mathrm{G}$ exponents $m$ and $z$ in terms of two unknown parameters, $\alpha$ and $\beta$. Our theoretical expressions for the $\mathrm{H}-\mathrm{G}$ exponents can be written as

$$
\begin{aligned}
b & =1 / 2 \\
z & =\left(1-3 / D_{\mathrm{T}}\right) /(3 \alpha-2 \beta) \\
m & =\left(z \alpha+1 / D_{\mathrm{T}}\right) / 2 \\
f & =1 / 2-m .
\end{aligned}
$$

\subsection{Horton law for the Manning friction and a theoretical expression for its exponent}

The scaling exponents $\alpha$ and $\beta$ are free parameters, which are not predicted by our theory. As a result, $m, f=1 / 2-m$ and $z$ are not predicted and tested against data in this paper. This poses a big challenge for testing our results based on an application of SS-2. To make progress with this necessary component of the theory, we consider the Manning friction coefficient that can be estimated from the observed values of depth and slope. We derive a theoretical expression for the Manning friction exponent here, and test it against the data in the next subsection.

Rewrite $\Pi_{6}^{*}(\omega)$ given by Eq. (36) as

$\Pi_{6}^{*}(\omega)=\frac{U_{\omega}^{2}}{g D_{\omega} S_{\omega}^{\beta}}=\frac{U_{\omega}^{2}}{g D_{\omega} S_{\omega} S_{\omega}^{-1+\beta}}$,

so that it may be expressed in the form of the well-known Chezy equation:

$U_{\omega}=C_{\omega}^{*} \sqrt{D_{\omega} S_{\omega}}=\left(g D_{\omega} S_{\omega}\right)^{1 / 2} S_{\omega}^{(-1+\beta) / 2}\left[\Pi_{6}^{*}(\omega)\right]^{1 / 2}$.

Therefore, an expression for the Chezy friction parameter is given as

$C_{\omega}^{*}=\left[\Pi_{6}^{*}(\omega)\right]^{1 / 2}(g)^{1 / 2} S_{\omega}^{(-1+\beta) / 2}$.

Since the slope ratio converges to the Horton number $R_{S}$, and ratio $R_{\Pi_{6}^{*}}$ converges to 1 so does Chezy friction parameter. Moreover, $\beta<1$ is a physical constraint, because the slope exponent must be negative to be consistent with the data. The Manning friction coefficient $n_{\omega}^{\prime}$ is related to Chezy's as follows (Leopold et al., 1964, p. 158):

$U_{\omega}=C_{\omega}^{*} \sqrt{D_{\omega} S_{\omega}}=\frac{1.49}{n_{\omega}^{\prime}} D_{\omega}^{2 / 3} S_{\omega}^{1 / 2}=\frac{1.49}{n_{\omega}^{\prime}} D_{\omega}^{1 / 6} \sqrt{D_{\omega} S_{\omega}}$.
Therefore,

$n_{\omega}^{\prime}=1.49 D_{\omega}^{1 / 6} / C_{\omega}^{*}$.

It can be expressed as a ratio:

$$
\frac{C_{\omega+1}^{*}}{C_{\omega}^{*}}=\left[\frac{D_{\omega+1}}{D_{\omega}}\right]^{1 / 6}\left[\frac{n_{\omega}^{\prime}}{n_{\omega+1}^{\prime}}\right] .
$$

Substituting Eqs. (48) and taking the limit as $\Omega-\omega \rightarrow \infty$ gives

$R_{n^{\prime}}=R_{D}^{1 / 6} R_{S}^{-(-1+\beta) / 2}$.

Using the definition of $n^{\prime}$ from Eq. (7) and applying the result from Eq. (21) gives $R_{n^{\prime}}=R_{Q}^{y}$. Using $R_{S}=R_{Q}^{z}$ as before gives an expression for the $\mathrm{H}-\mathrm{G}$ scaling exponent related to the Manning equation,

$R_{n^{\prime}}=R_{Q}^{y}=R_{Q}^{f / 6} R_{Q}^{-z(-1+\beta) / 2}$,

which gives a theoretical prediction for the Manning friction exponent as

$y=f / 6-z(-1+\beta) / 2$.

There are no free parameters in this expression once $\beta$ is estimated as explained in the next subsection, where this prediction is tested. The constraints on estimated $\beta<1$, and $\beta<3 \alpha / 2$ from Eq. (44) are also tested there.

Mantilla et al. (2006) have described the $\mathrm{H}-\mathrm{G}$ form of the Chezy friction coefficient that they deduced from empirical observations. They showed that the expression for the Chezy friction coefficient played a key role in testing the presence of statistical self-similarity involving Horton laws in peak flows in the Walnut Gulch basin, Arizona.

\subsection{Test of the Manning scaling exponent for three field studies}

We predict the Manning exponent $y$ by using the empirical values of $f$ and $z$ for the three field studies described in Sect. 3, and test our prediction against the empirical values of $y$ as a second test of our theory. Despite the appearance of $D_{\mathrm{T}}$ in Eq. (45) and thereby in Eq. (53), and the regularity of its observed values between 1.7 and 1.8 (La Barbera and Rosso, 1989), given $f$ and $z$, the exponent $\beta$ does not depend on $D_{\mathrm{T}}$; i.e., our test becomes independent of the fractal dimension of the channel network. To see this feature, notice from Eqs. (45) that one gets after straightforward algebra, $\beta=(1-3 f) / z$. Therefore, $y$ can be rewritten strictly as a function of $f$ and $z$,

$y=f / 6-z(-1+\beta) / 2=5 f / 3+z / 2-1 / 2$.

The above argument does not imply that the set of $\mathrm{H}-\mathrm{G}$ exponents is independent of $D_{\mathrm{T}}$. As Eq. (45) shows, the depth and 
slope exponents $f$ and $z$ depend on $\alpha, \beta$ and $D_{\mathrm{T}}$. Therefore, the influence of $D_{\mathrm{T}}$ on $y$ comes through $f$ and $z$.

The first test of the theory is for the Brandywine creek (Leopold et al., 1964, Table 7.5, p. 244). The observed values of the $\mathrm{H}-\mathrm{G}$ exponents were given in Sect. 3 and in Table 1 . Equation (54) correctly predicts the empirical value of the Manning exponent, $y=-0.285$. This prediction also supports our assumption made in Sect. 6.2 that the observed depth and velocity exponents are correct, because they lead to a correct prediction of the Manning exponent.

The second test is conducted in the Taieri River basin. Ibbitt et al. (1998) do not give an empirical value of Manning's friction exponent, but it can be computed from the empirical Manning equation using the exponents $f$ and $z$. We first use the values of the exponents given Sect. 3, and then incorporate the errors in these exponents to compute an interval for $y$. The exponents along with the errors are listed in Table 1 . Using the Manning equation,

$U_{\omega}=1.49 D_{\omega}^{2 / 3} S_{\omega}^{1 / 2} / n_{\omega}^{\prime}$,

the observed exponent $y$ is given by

$y=(2 / 3)(0.247)+(1 / 2)(-0.315)-0.238=-0.231$.

The measurement errors in the exponents listed in Table 1 give the range $(-0.259,-0.203)$ for $y$.

To make a theoretical prediction of $y$, using the empirically computed values of the scaling exponents $f$ and $z$, the predicted value of the Manning exponent from Eq. (54) is -0.246 . The measurement errors in the exponents $f$ and $z$ listed in Table 1 lead to the prediction of the range $(-0.276$, $-0.216)$. This predicted interval overlaps with the empirical interval of $y$ computed above. It supports our prediction in the Taieri basin.

The third field test is for the Ashley River basin. The exponents are given in Sect. 3 and in Table 1. McKerchar et al. (1998) do not give an empirical value of the Manning exponent, but it can be computed from observed exponents $z$ and $f$ and the empirical Manning equation. The value is

$y=(2 / 3)(0.242)-0.318-(1 / 2)(0.317)=-0.315$.

Using the observed values of $f$ and $z$, the predicted value of the Manning exponent using Eq. (54) is

$y=5(0.242) / 3+(-0.317) / 2-1 / 2=-0.255$.

The measurement errors in the exponents give the range for the empirical exponent $y$ as $(-0.338,0.292)$. Using the observed values with errors in $f$ and $z$, the range of the predicted scaling exponent $y$ is $(-0.281,-0.230)$. Clearly, the predicted interval does not overlap with the empirical interval. There is some discrepancy between the observed and predicted values. It seems to come from the observed exponents of width and velocity. The discrepancy in the width exponent noted in Sect. 6.2 affects the velocity exponent, which in turn affects the depth exponent and the Manning friction exponent. This important topic is the focus of the next section.

Table 1 presents a summary of the observed and predicted $\mathrm{H}-\mathrm{G}$ scaling exponents for the three basins considered above. Predicted values for the exponent $y$ from Eq. (53), for the exponent $b=1 / 2$ from Eq. 27 , and for the exponent $m+f=$ $1 / 2$ from Eq. 28 are given in Table 1 .

\subsection{Test of Horton laws and the $R_{Q}=R_{A}$ relationship for the Ashley basin}

Mantilla (2014) is testing the presence of Horton laws for the $\mathrm{H}-\mathrm{G}$ variables in the two NZ basins considered here and a few other basins like the Whitewater one for which he has data. He has kindly shared some of his analyses with us for the Ashley basin, which has $\Omega=6$. He extracted the Ashley basin geomorphology from the digital elevation model (DEM) data using the CUENCAS software (Mantilla and Gupta, 2005). His first set of results pertains to the Horton laws for drainage area and stream numbers as shown in Figs. 3 and 4. The Horton laws hold quite well, and the observed $R_{A}=4.47$ agrees well with $R_{B}=4.5$ as predicted for the Tokunaga network in Sect. 2.1.

Next, the Horton law for the width is shown in Fig. 5. In making this plot, the theoretical condition $\Omega-\omega \rightarrow \infty$ is incorporated by omitting order 6 and 5 streams from the analysis. Mantilla (2014) found that the basin has a large number of the 1st order streams that are mostly missed in the map that McKerchar et al. (1998) presented. Therefore, the Horton plot is made for streams of order $\omega=2,3,4$, shown in Fig. 5. Horton width ratio, $R_{W}=1.61$ is observed. Other $\mathrm{H}-$ $\mathrm{G}$ variables not shown here support the finding that the Horton laws for the $\mathrm{H}-\mathrm{G}$ variables hold for the Ashley basin, as predicted in our work.

Next, mean stream flow is considered. Similar to the width, a Horton plot is made for streams of order $\omega=2,3,4$ as shown in Fig. 6. $R_{Q}=3.05$ is observed. As a test of Eq. (22), we note using the observed width exponent that $3.05^{0.44}=1.63$ is consistent with the observed value of $R_{W}=1.61$ that Mantilla (2014) obtained. Other $\mathrm{H}-\mathrm{G}$ variables not shown here support that the Horton laws hold as predicted in our work, and $R_{Q}=R_{W} R_{D} R_{U}=2.96$ is close to the value of $R_{Q}=3.05$. We do not include errors given in Table 1 to simplify our argument. These results demonstrate the observed consistency between the scaling exponents and the Horton ratios for the $\mathrm{H}-\mathrm{G}$ variables that are derived in Sect. 6.

The next task is to test if the $R_{Q}=R_{A}$ given by Eq. 18 holds in the Ashley basin. Data show that $\log R_{Q} / \log R_{A}=$ 0.74 . It can be written as

$R_{Q}=R_{A}^{\theta}$,

where $\theta=0.74$. Clearly, our theory's prediction that $R_{Q}$ equals $R_{A}$ given in Eq. (18) is not supported by this 


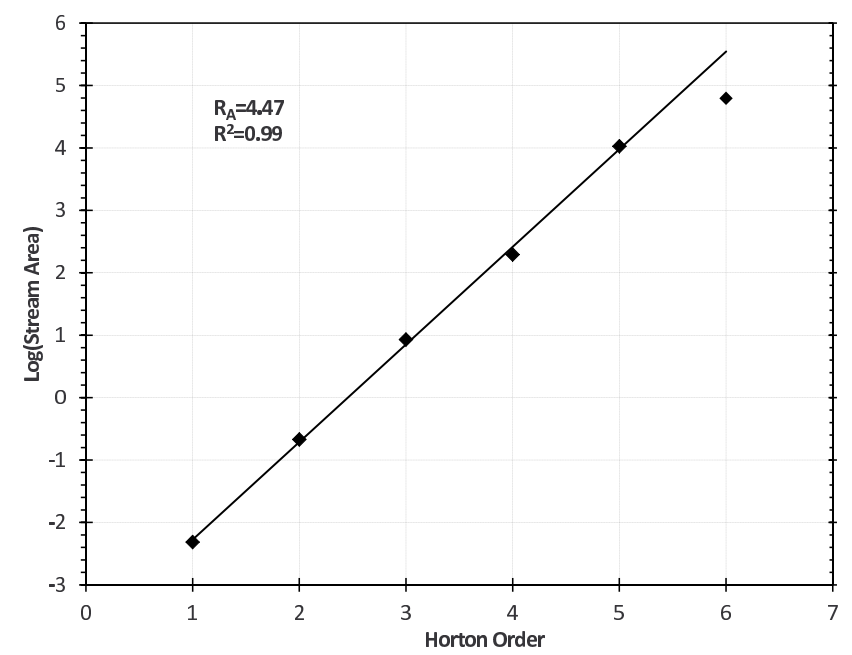

Figure 3. Horton analysis of upstream areas (including orders 2, 3, and 4) for the Ashley River basin (McKerchar et al., 1998); results kindly provided by Mantilla (2014).

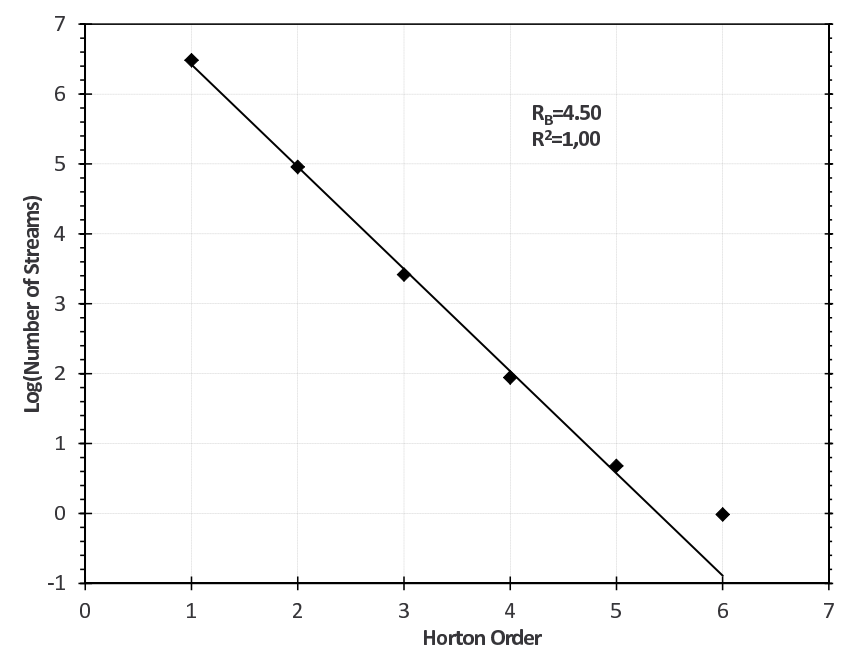

Figure 4. Horton analysis of stream numbers (including orders 2, 3, and 4) for the Ashley River basin (McKerchar et al., 1998); results kindly provided by Mantilla (2014).

observation. The physical reason is that all the streams in a network do not contribute to stream flows in the Ashley basin. Many physical processes play a role in runoff generation, like space-time variable rainfall, the state of dryness or wetness of the soil in a basin at the time rainfall begins, which governs infiltration into soil and evaporation from it, and so on. The physical parameter, $\theta$, represents the aggregate behavior of the physical processes governing runoff generation, and can take a value less than or equal to 1 .

Galster (2007) analyzed several basins to test the relationship $Q=k A^{c}$. His results show that the studied watersheds could be grouped into two broad categories based on their respective $c$ values: (1) those where c equals 1 or nearly 1 ,

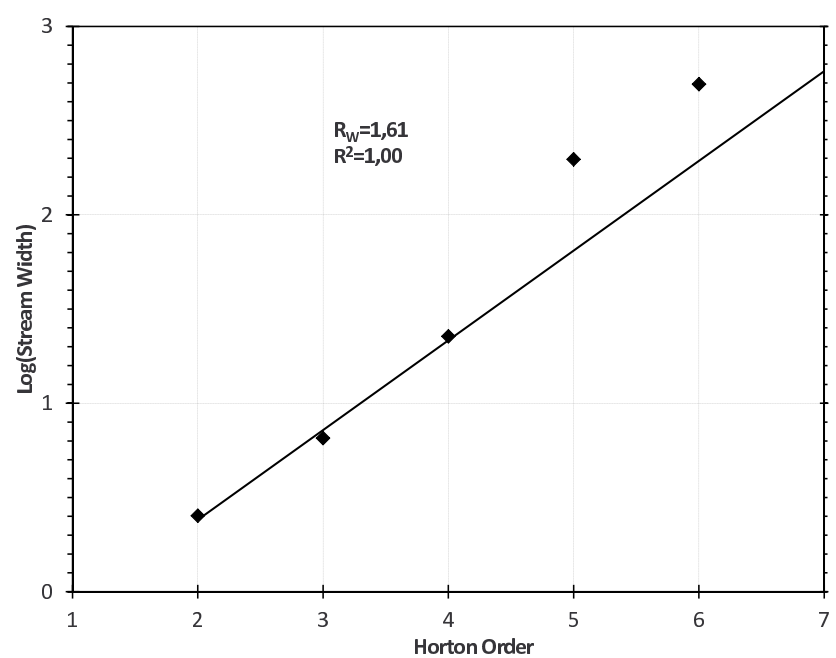

Figure 5. Horton plot for stream width (including orders 2, 3, and 4) for the Ashley river basin (McKerchar et al., 1998); results kindly provided by Mantilla (2014).

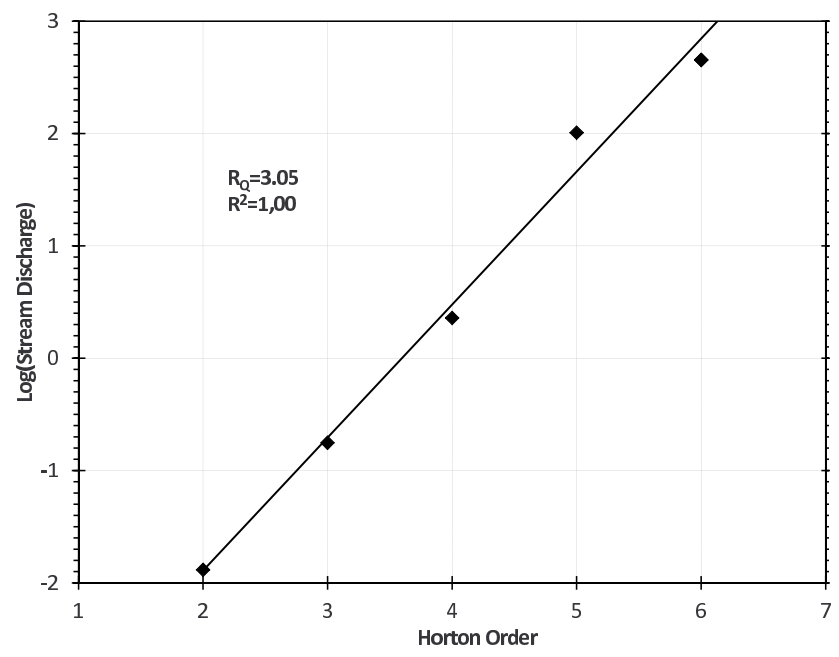

Figure 6. Horton plot for discharge (including orders 2, 3, and 4) for the Ashley River basin (McKerchar et al., 1998); results kindly provided by Mantilla (2014).

and (2) those where $c$ is significantly less than 1 , like 0.8 or 0.5 . The reader may note that our exponent $\theta$ is equivalent to $c$. Moreover, the derivation in Eq. (18) is that $R_{Q}=R_{A}$ applies to category (1) basins in Galster (2007), but not to category (2) basins like Ashley. Therefore, our theory needs to be generalized to incorporate such basins for which $\theta$ is less than 1 .

\section{Future research problems: two examples}

The above theory can be generalized along several lines. We illustrate two important problems. The first is that the anomalous scaling exponents $\alpha$ and $\beta$ need to be predicted 
using physical arguments. Two fundamental physical processes that shape the $\mathrm{H}-\mathrm{G}$ of channels are transport of suspended sediment load and the bed load that we have not considered so far. There is a vast literature on this subject (Leopold et al., 1964; Singh, 2003). Our ideas on how these two physical processes can be used to determine $\alpha$ and $\beta$ are rudimentary and are only meant for illustration.

The suspended load increases in proportion to discharge. Therefore, suspended sediment concentration, defined as the ratio of the two, does not change. Leopold et al. (1964, p. 269) gave an expression for sediment concentration, $C \propto$ $(U D)^{0.5} S^{1.5} / n^{4}$. The constancy of $C$ implies that $0.5 m+$ $0.5 f+1.5 z-4 y=0$ or $0.25+1.5 z-4 y=0$, since $m+f=$ $1 / 2$. It gives the first equation in terms of $\alpha$ and $\beta$.

The second equation can be developed from considering stream power per unit of bed area, $\varpi=\rho g Q S / W$, which plays a basic role in the bed load transport (Molnar, 2001). Essentially all the theories of bed load transport assume that there is a threshold shear stress, stream power, or mean flow speed, and no erosion occurs below it. During floods, these variables exceed the threshold, and bed load is transported that creates erosion. We expect that a second equation can be obtained from these considerations in terms of $\alpha$ and $\beta$. The two equations can be solved to compute $\alpha$ and $\beta$.

Traditionally, Horton laws have been known in terms of statistical means. Peckham and Gupta (1999) reformulated the Horton laws in terms of probability distributions and called them "generalized Horton laws". Specifically, they gave observational and some theoretical arguments to show that probability distributions of all drainage areas rescaled by their means, $A_{\omega} / \bar{A}_{\omega}$ collapse into a common probability distribution. Let us consider drainage areas, $A_{\omega} / \bar{A}_{\omega}$ to illustrate generalized Horton laws. There are two components to this argument.

i. A Horton law for the mean drainage areas, $\bar{A}_{\omega}$ of order $\omega$, holds that can be written as

$\bar{A}_{\omega}=R_{A}^{\omega-1} \bar{A}_{1}, \omega=1,2, \ldots$,

where $R_{A}$ is the Horton area ratio. It is illustrated in the Whitewater basin, Kansas, USA in Fig. 7.

ii. A generalized Horton law is defined as

$$
A_{\omega+1} / \bar{A}_{\omega+1} \stackrel{d}{=} A_{\omega} / \bar{A}_{\omega}
$$

or

$$
A_{\omega+1} \stackrel{d}{=}\left(\bar{A}_{\omega+1} / \bar{A}_{\omega}\right) A_{\omega}, \omega=1,2, \ldots,
$$

where $\stackrel{d}{=}$ means that the probability distributions of the rescaled areas on both sides of Eq. (60) are the same. Since the Horton law holds for the mean areas given in Eq. (59), it follows from Eq. (61) that

$$
A_{\omega+1} \stackrel{d}{=} R_{A} A_{\omega}, \omega=1,2, \ldots
$$

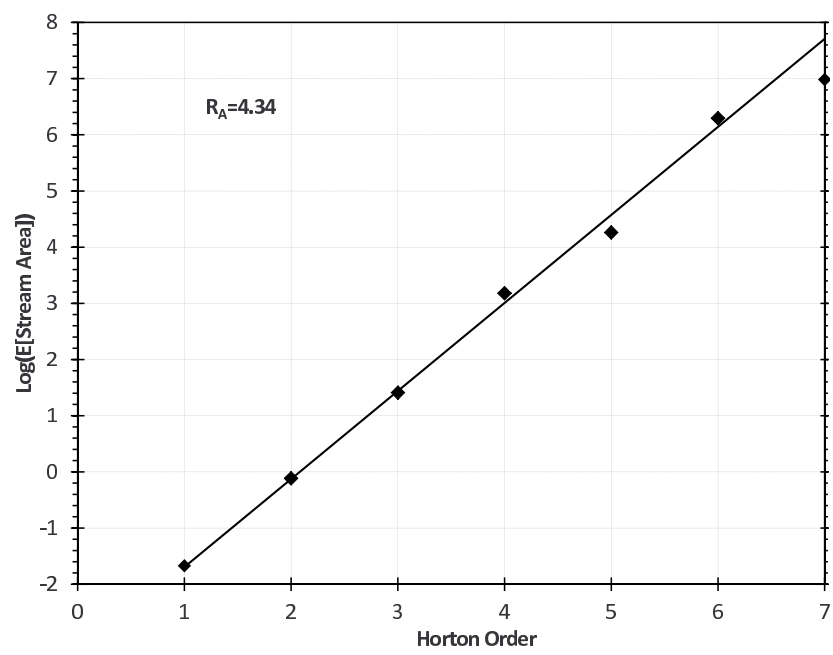

Figure 7. Reproduction of the original figure of Mantilla and Gupta (2005, Fig. 2) showing the scaling of mean drainage area with order (Horton law) of the river network of the Whitewater basin, Kansas, US.

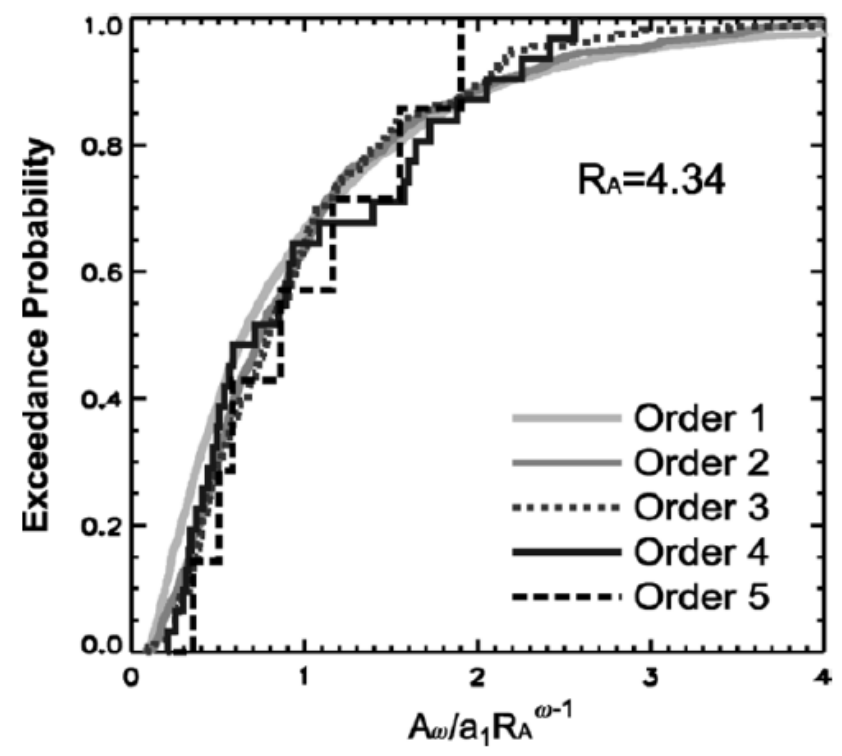

Figure 8. Reproduction of the original figure of Mantilla and Gupta (2005, Fig. 2) showing the statistical scaling of the probability distribution of drainage area with order (generalized Horton law) of the river network in the Whitewater basin, Kansas, US.

Mantilla and Gupta (2005) have shown the existence of generalized Horton law for the rescaled drainage areas, $A_{\omega} / \bar{A}_{\omega}$, because it is independent of $\omega$. This feature is illustrated for the Whitewater basin, Kansas, USA in Fig. 8.

Let us consider the dependence of channel widths on discharge. Both are treated as random variables. Therefore, the results obtained in Sect. 6.2 can be interpreted as those for the means and written as $\bar{W}\left(Q_{\omega}\right)=c \bar{Q}_{\omega}^{b}$. We conjecture that 
the generalized Horton law holds for the rescaled channel widths, and write it as

$$
W\left(Q_{\omega+1}\right)=\left(\frac{\bar{Q}_{\omega+1}}{\bar{Q}_{\omega}}\right)^{b} W\left(Q_{\omega}\right), \omega=1,2, \ldots
$$

This is an equality between random variables, as shown for drainage areas in Eq. (61). It means that the probability distribution of $W\left(Q_{\omega+1}\right)$ can be computed from the probability distribution of $W\left(Q_{\omega}\right)$ provided a Horton law of mean widths and the value of $b$ are known. Both these features are predicted in Sect. 6 for self-similar Tokunaga networks. Our conjecture is made in the light of the result that the Tokunaga networks are a special case of a subclass of RSN (Veitzer and Gupta, 2000). In view of these arguments, we can write

$\bar{W}_{\omega}=R_{W}^{\omega-1} \overline{W_{1}}, \quad \omega=1,2, \ldots$,

where $R_{W}=R_{Q}^{b}$ is the Horton ratio for the mean widths. We conjecture based on these arguments that Horton laws hold for all the $\mathrm{H}-\mathrm{G}$ variables measured in the two New Zealand basins that were analyzed in Sect. 8.3. We supported our conjecture for the validity of Horton laws for widths and stream flows in the Ashley basin in Sect. 8.3. Both the NZ basins have the necessary data sets to test our conjecture regarding the applicability of the Horton laws and the generalized Horton laws for all the $\mathrm{H}-\mathrm{G}$ variables considered in this paper. Mantilla (2014) is conducting this research.

\section{Conclusions}

There has been important progress in topological and geometric theories to explain the related Horton law for stream bifurcation, drainage areas and stream lengths as asymptotic relations, but progress on Horton laws for the $\mathrm{H}-\mathrm{G}$ variables has been long overdue. We made a contribution to this important problem, and laid the theoretical foundations of a $\mathrm{H}-\mathrm{G}$ theory in the self similar Tokunaga networks. Our main findings are summarized below:

1. The theory used several disjoint theoretical concepts like Horton laws of stream numbers and areas as asymptotic relations in Tokunaga networks, dimensional analysis, the Buckingham Pi theorem, SS-1 and SS-2. A self-contained review of these concepts with examples was given as "methods" in Sect. 2.

2. The $\mathrm{H}-\mathrm{G}$ data sets in channel networks from three published studies and one unpublished study were summarized for testing theoretical predictions in Sect. 3.

3. An important issue in estimating field values of the H$\mathrm{G}$ exponents is the measurement errors, which affect the scaling exponents. Standard errors for each of the $\mathrm{H}-\mathrm{G}$ exponents from the two NZ basins are listed in Table 1.
4. We used the Buckingham Pi theorem and identified six dimensionless basin numbers in Sect. 4, which served as a basis for developing the theory in the subsequent sections.

5. A mass conservation equation was specified in Strahler ordered networks. A link-based equation as a special case of our equation has been known (Gupta et al., 2007). We solved it in Tokunaga networks using the results from McConnell and Gupta (2008), and derived a mass conservation equation in terms of Horton bifurcation and the discharge ratios in the limit as $\Omega-\omega$ goes to infinity in Sect. 5. We also derived that the Horton discharge ratio is equal to the area ratio.

6. We gave an analytical derivation of the $\mathrm{H}-\mathrm{G}$ relations as power-law functions of discharge. The derivation is based on the assumptions that the $\mathrm{H}-\mathrm{G}$ variables are homogeneous and self-similar functions of discharge. The Horton laws are extended to width, depth and velocity in Tokunaga networks using the results from Sect. 5. Within the dimensional analysis framework, the SS-1 given in Barenblatt (1996) is used to predict the width exponent, $b=1 / 2$, and the Reynolds number exponent $m+f=1 / 2$. These predictions are tested against data sets given in Sect. 3 including the standard errors listed in Table 1. These results are given in Sect. 6.

7. Tentatively assuming that SS-1 holds for slope, we predicted the Horton laws for $S, U$ and $D$, and their exponents. Our predictions agree with the exponents given in the optimal channel network (OCN) model (RodríguezIturbe et al., 1992), but OCN does not consider Horton laws. Our theoretical framework is based on selfsimilarity, and does not use any optimality assumptions. The published data in Sect. 3 showed that most of the OCN predictions do not agree with observations. These results are given in Sect. 7.

8. SS-2 is required to deal with the case when one or more dimensionless numbers go to zero in the limit, but they cannot be ignored in the limit. In the present context, slope goes to zero in the limit of large basin order, but it cannot be ignored. Therefore, SS-2 is needed, which gives rise to two anomalous scaling exponents, $\alpha$ and $\beta$ that come from two dimensionless numbers in Sect. 4. We derived Horton's law for $S, D$, and $U$ in Sect. 8, but the $\mathrm{H}-\mathrm{G}$ exponents become functions of $\alpha$ and $\beta$.

9. We do not give a physical prediction of $\alpha$ and $\beta$. To make progress with testing our theory, the Manning friction exponent $y$ is considered because it could be estimated from data on slope, velocity, width and depth, as well as predicted from our theory using the observed exponents for $D$ and $S$. Standard errors in the exponents are considered in carrying out these tests of the theory. The predictions are excellent for two of the three basins, 
but show some discrepancy in the Ashley basin, given in Sect. 8.3.

10. The validity of the Horton laws in the Ashley basin was tested using results from the study by Mantilla (2014) that is in progress, but we showed some results for the existence of Horton laws for widths and stream flows.

11. The test of $R_{Q}=R_{A}$ showed that this prediction does not hold in the Ashley basin, because some of the assumptions leading to Eq. (17) do not hold. To incorporate this hydrologic feature into Tokunaga networks, the generator expression given in Eq. (2) needs to be modified so that all the streams that do not contribute to stream flows are removed in the derivation of Eq. (17).

12. The estimation of the anomalous exponents from physical principles using considerations of sediment transport are briefly discussed. Further development is needed on this front for a definite test of the theory.

13. Two NZ basins analyzed here show statistical variability in the $\mathrm{H}-\mathrm{G}$ variables that is different from the measurement errors. Generalized Horton laws are explained and illustrated for the Whitewater basin, Kansas, USA. It needs to be tested for the $\mathrm{H}-\mathrm{G}$ variables for a further development of this theory. This important research is in progress (Mantilla, 2014).

Acknowledgements. We are grateful to Ricardo Mantilla, Iowa Flood Center, University of Iowa, for sharing his data analysis for this paper. Peckham (1995b) briefly explored the idea of using dimensional analysis for determining Horton ratios. However, there were too many loose ends at the time to make further progress. This project has taken more than a decade and a half to get the results presented here. It is far beyond the duration of a single or several NSF grants. However, support from NSF kept one of us working on scaling and self-similarity ideas in floods, where $\mathrm{H}-\mathrm{G}$ is required to model flow dynamics in a channel network. Vijay Gupta gratefully acknowledges this support. Oscar Mesa acknowledges continuous support from Universidad Nacional de Colombia and Colciencias research project 502/2010.

Edited by: R. Donner

Reviewed by: two anonymous referees

\section{References}

Barenblatt, G. I.: Scaling, self-similarity, and intermediate asymptotics, Cambridge University Press, Cambridge, UK, 1996.

Barenblatt, G. I.: Scaling, vol. 34, Cambridge University Press, Cambridge, UK, 2003.

Berry, M. and Bradley, P.: The application of network analysis to the study of branching patterns of large dendritic fields, Brain Research, 109, 111-132, 1976.

Borchert, R. and Slade, N. A.: Bifurcation ratios and the adaptive geometry of trees, Bot. Gaz., 142, 394-401, 1981.
Clayton, J. and Kean, J.: Establishing a Multi-scale Stream Gaging Network in the Whitewater River Basin, Kansas, USA, Water Resour. Manag., 24, 3641-3664, doi:10.1007/s11269-010-9624$\mathrm{x}, 2010$.

Dawdy, D. R.: Prediction versus understanding (The 2006 Ven Te Chow Lecture), J. Hydrol. Eng., 12, 1-3, 2007.

Dawdy, D. R., Griffis, V. W., and Gupta, V. K.: Regional floodfrequency analysis: How we got here and where we are going, J. Hydrol. Eng., 17, 953-959, 2012.

Dodds, P. S. and Rothman, D. H.: Unified view of scaling laws for river networks, Phys. Rev. E, 59, 4865, doi:10.1103/PhysRevE.59.4865, 1999.

Furey, P. R. and Gupta, V. K.: Space-time variability of low streamflows in river networks, Water Resour. Res., 36, 2679-2690, doi:10.1029/2000WR900136, 2000.

Furey, P. R., Gupta, V. K., and Troutman, B. M.: A top-down model to generate ensembles of runoff from a large number of hillslopes, Nonlin. Processes Geophys., 20, 683-704, doi:10.5194/npg-20-683-2013, 2013.

Galster, J. C.: Natural and anthropogenic influences on the scaling of discharge with drainage area for multiple watersheds, Geosphere, 3, 260-271, 2007.

Gibbings, J.: Dimensional analysis, Springer London, doi:10.1007/978-1-84996-317-6, 2011.

Gupta, V. K. and Waymire, E.: Spatial variability and scale invariance in hydrologic regionalization, in: Scale dependence and scale invariance in hydrology, edited by: Sposito, G., Cambridge University Press, London, 88-135, 1998.

Gupta, V. K., Troutman, B. M., and Dawdy, D. R.: Towards a nonlinear geophysical theory of floods in river networks: an overview of 20 years of progress, in: Nonlinear dynamics in geosciences, edited by: Tsonis, A. A. and Elsner, J. B., Springer, New York, NY 10013, USA, 121-151, doi:10.1007/978-0-38734918-3_8, 2007.

Gupta, V. K., Mantilla, R., Troutman, B. M., Dawdy, D., and Krajewski, W. F.: Generalizing a nonlinear geophysical flood theory to medium-sized river networks, Geophys. Res. Lett., 37, L11402, doi:10.1029/2009GL041540, 2010.

Horsfield, K.: Are diameter, length and branching ratios meaningful in the lung?, J. Theor. Biol., 87, 773-784, 1980.

Horton, R. E.: Erosional development of streams and their drainage basins; hydrophysical approach to quantitative morphology, Geol. Soc. Am. Bull., 56, 275-370, 1945.

Ibbitt, R., McKerchar, A., and Duncan, M.: Taieri River data to test channel network and river basin heterogeneity concepts, Water Resour. Res., 34, 2085-2088, 1998.

Jarvis, R. S. and Woldenberg, M. J.: River networks, vol. 80, Hutchinson Ross, New York, USA, 1984.

Kean, J. W. and Smith, J. D.: Generation and verification of theoretical rating curves in the Whitewater River basin, Kansas, J. Geophys. Res., 110, F04012, doi:10.1029/2004JF000250, 2005.

Köppen, W.: Die Wärmezonen der Erde, nach der Dauer der heissen, gemässigten und kalten Zeit und nach der Wirkung der Wärme auf die organische Welt betrachtet, Meteorologische Zeitschrift, 1, 215-226, translated and edited by: Volken, E. and Brönnimann, S., The thermal zones of the Earth according to the duration of hot, moderate and cold periods and to the impact of heat on the organic world, Meteorol. Z., 20, 351-360, 1884. 
La Barbera, P. and Rosso, R.: On the fractal dimension of stream networks, Water Resour. Res., 25, 735-741, 1989.

Leopold, L. and Maddock, T.: The Hydraulic Geometry of Stream Channels and Some Physiographic Implications, USGS Professional Paper: 252, 57 pp., 1953.

Leopold, L. and Miller, J.: Ephemeral streams: Hydraulic factors and their relation to the drainage net, USGS Professional Paper: 282-A, 37 pp., 1956.

Leopold, L., Wolman, M. G., and Miller, J. P.: Fluvial processes in geomorphology, WH Freeman, San Francisco, 1964.

Lima, C. H. and Lall, U.: Spatial scaling in a changing climate: A hierarchical bayesian model for non-stationary multi-site annual maximum and monthly streamflow, J. Hydrol., 383, 307318, 2010.

Mantilla, R.: Physical basis of statistical scaling in peak flows and stream flow hydrographs for topologic and spatially embedded random self-similiar channel networks, Ph.D. thesis, University of Colorado, Boulder, USA, 2007.

Mantilla, R.: Statistical Self-Similarity in Ordered Networks for Hydraulic-Geometric variables in river basins, unpublished manuscript, 2014.

Mantilla, R. and Gupta, V. K.: A GIS numerical framework to study the process basis of scaling statistics in river networks, Geoscience and Remote Sensing Letters, IEEE, 2, 404-408, 2005.

Mantilla, R., Gupta, V. K., and Mesa, O. J.: Role of coupled flow dynamics and real network structures on Hortonian scaling of peak flows, J. Hydrol., 322, 155-167, 2006.

Maritan, A., Colaiori, F., Flammini, A., Cieplak, M., and Banavar, J. R.: Universality Classes of Optimal Channel Networks, Science, 272, 984-986, doi:10.1126/science.272.5264.984, 1996.

McConnell, M. and Gupta, V. K.: A proof of the Horton law of stream numbers for the Tokunaga model of river networks, Fractals, 16, 227-233, 2008.

McKerchar, A., Ibbitt, R., Brown, S., and Duncan, M.: Data for Ashley River to test channel network and river basin heterogeneity concepts, Water Resour. Res., 34, 139-142, 1998.

Molnar, P.: Climate change, flooding in arid environments, and erosion rates, Geology, 29, 1071-1074, 2001.

Molnar, P. and Ramirez, J. A.: On downstream hydraulic geometry and optimal energy expenditure: case study of the Ashley and Taieri Rivers, J. Hydrol., 259, 105-115, 2002.

Ogden, F. L. and Dawdy, D. R.: Peak discharge scaling in small Hortonian watershed, J. Hydrol. Eng., 8, 64-73, 2003.

Park, D.: Does Horton's law of branch length apply to open branching systems?, J. Theor. Biol., 112, 299-313, 1985.

Peckham, S. D.: New results of self-similar trees with applications to river networks, Water Resour. Res., 31, 1023-1029, 1995a.

Peckham, S. D.: Self-similarity in the three-dimensional geometry and dynamics of large river basins, Ph.D. thesis, University of Colorado, Boulder, USA, 1995b.

Peckham, S. D. and Gupta, V. K.: A reformulation of Horton's laws for large river networks in terms of statistical self-similarity, Water Resour. Res., 35, 2763-2777, 1999.
Poveda, G., Vélez, J. I., Mesa, O. J., Cuartas, A., Barco, J., Mantilla, R. I., Mejía, J. F., Hoyos, C. D., Ramírez, J. M., Ceballos, L. I., Zuluaga, M. D., Arias, P. A., Botero, B. A., Montoya, M. I., Giraldo, J. D., and Quevedo, D. I.: Linking long-term water balances and statistical scaling to estimate river flows along the drainage network of Colombia, J. Hydrol. Eng., 12, 4-13, doi:10.1061/(ASCE)1084-0699(2007)12:1(4), 2007.

Pries, A. R. and Secomb, T. W.: Blood Flow in Microvascular Networks, Comprehensive Physiology, doi:10.1002/cphy.cp020401, online first, 2011.

Rodríguez-Iturbe, I., Rinaldo, A., Rigon, R., Bras, R. L., Marani, A., and Ijjász-Vásquez, E.: Energy dissipation, runoff production, and the three-dimensional structure of river basins, Water Resour. Res., 28, 1095-1103, 1992.

Rudin, W.: Principles of mathematical analysis, McGraw Hill, New York, USA, 3rd Edn., 1976.

Shreve, R. L.: Infinite topologically random channel networks, J. Geol., 75, 178-186, 1967.

Singh, V. P.: On the theories of hydraulic geometry, Int. J. Sediment Res., 18, 196-218, 2003.

Sivapalan, M., Takeuchi, K., Franks, S. W., Gupta, V. K., Karambiri, H., Lakshmi, V., Liang, X., McDonnell, J. J., Mendiondo, E. M., O'connell, P. E., Oki, T., Pomeroy, J. W., Schertzer, D., Uhlenbrook, S., and Zehe, E.: IAHS Decade on Predictions in Ungauged Basins (PUB), 2003-2012: Shaping an exciting future for the hydrological sciences, Hydrolog. Sci. J., 48, 857880, 2003.

Strahler, A. N.: Hypsometric (area-altitude) analysis of erosional topography, Geol. Soc. Am. Bull., 63, 1117-1142, 1952.

Strahler, A. N.: Quantitative analysis of watershed geomorphology, Eos T. Am. Geophys. Un., 38, 913-920, doi:10.1029/TR038i006p00913, 1957.

Tokunaga, E.: Consideration on the composition of drainage networks and their evolution, Geographical reports of Tokyo Metropolitan University, 13, 1-27, 1978.

Troutman, B. M.: Scaling of flow distance in random self-similar channel networks, Fractals, 13, 265-282, 2005.

Veitzer, S. and Gupta, V. K.: Random self-similar river networks and derivations of generalized Horton laws in terms of statistical simple scaling, Water Resour. Res., 36, 1033-1048, 2000.

Veitzer, S. A., Troutman, B. M., and Gupta, V. K.: Power-law tail probabilities of drainage areas in river basins, Phys. Rev. E, 68, 016123, doi:10.1103/PhysRevE.68.016123, 2003.

Viennot, G. and Vauchaussade de Chaumont, M.: Enumeration of RNA Secondary Structures by Complexity, in: Mathematics in Biology and Medicine, edited by: Capasso, V., Grosso, E., and Paveri-Fontana, S., Lect. Notes Biomath., Springer Berlin Heidelberg, 57, 360-365, doi:10.1007/978-3-642-93287-8_50, 1985.

West, G. B., Brown, J. H., and Enquist, B. J.: A general model for the origin of allometric scaling laws in biology, Science, 276, 122-126, 1997. 